\title{
KRONECKER CONSTANTS FOR FINITE SUBSETS OF INTEGERS
}

\author{
KATHRYN E. HARE AND L. THOMAS RAMSEY
}

\begin{abstract}
A set of integers $S$ is called $\varepsilon$-Kronecker if every function on $S$ of modulus one can be approximated uniformly to within $\varepsilon$ by a character. The least such $\varepsilon$ is called the $\varepsilon$-Kronecker constant.

We transform the problem of calculating $\varepsilon$-Kronecker constants for finite sets of $d$ elements into a geometric optimization problem. Using this approach we can explicitly determine the $\varepsilon$-Kronecker constant for any two element set and deduce a (non-trivial) upper bound for any finite set. Kronecker constants are determined for many classes of three element sets, including all sum sets, product sets and arithmetic progressions. The answers are surprisingly complicated.
\end{abstract}

\section{INTRODUCTION}

A subset $S$ of the dual of a compact, abelian group $G$ is called an $\varepsilon$-Kronecker set if for every continuous function $f$ mapping $S$ into $\mathbb{T}$, the set of complex numbers of modulo 1 , there exists $x \in G$ such that

$$
|\gamma(x)-f(\gamma)|<\varepsilon \text { for all } \gamma \in S \text {. }
$$

The infimum of such $\varepsilon$ is called the Kronecker constant, $\kappa(S)$.

$\varepsilon$-Kronecker sets were introduced by Varopoulos [Varopoulos 1968] and were called $\varepsilon$-free in [Givens and Kunen 2003]. The concepts were discussed in the Seminaire Bourbaki (1964-1966) without formal naming [Kahane 1995]. Sets whose Kronecker constants are zero have been much studied (c.f. [Graham and McGehee 1979] and the references cited therein), and are called Kronecker sets. Hadamard sets with ratio greater than two are $\varepsilon$-Kronecker subsets of $\mathbb{Z}$ for appropriate $\varepsilon<2$ ([Graham and Hare 2006a] or [Kunen and Rudin 1999]) and in many other groups infinite $\varepsilon$-Kronecker sets are known to exist for suitable (small) choices of $\varepsilon$ (please see [Galindo and Hernandez 1999] and [Graham and Lau 2007]).

The case when $\varepsilon<\sqrt{2}$ is of particular interest as such sets are Sidon, meaning every bounded function defined on the set $S$ is the restriction of the Fourier transform of a measure on $G .{ }^{1}$ In fact, the interpolating measure can be taken to be discrete (and even positive or supported on an open set under suitable assumptions) and $\sqrt{2}$ is sharp with this property ([Graham and Hare 2006a], [Graham and Hare 2003], [Graham and Hare 2006b]). Like Sidon sets, $\varepsilon$-Kronecker sets satisfy various arithmetic properties (c.f. [Graham, Hare and Korner 2006]).

1991 Mathematics Subject Classification. Primary: 42A15, 43A46, 65T40.

Key words and phrases. Kronecker set, interpolation, trigonometric approximation.

This research was supported in part by NSERC. The first author would like to thank the Dept. of Mathematics at University of Hawaii for their hospitality while this research was being done.

This paper is in final form and no version of it will be submitted for publication elsewhere.

${ }^{1}$ See [Lopez and Ross 1975] for basic facts about Sidon sets. 
All finite sets are $\varepsilon$-Kronecker, but relatively little is known about their Kronecker constants. In [Graham and Hare 2006a] an investigation of the Kronecker constant was begun for special examples of (mainly) subsets of integers of size two, but even for two-element sets the answers were very incomplete. One reason for the interest in studying finite sets is that the Kronecker constant of an infinite set is the supremum of the Kronecker constants of its finite subsets, as an easy compactness argument shows. Also, one can construct examples of infinite sets with interesting properties by 'piecing together' finite sets whose Kronecker constants are known. ([Graham and Hare 2006a, Ex. 5.2] is such an example.) Furthermore, Sidon sets can be characterized by the property that all of their finite subsets contain proportionally sized subsets that are $\varepsilon$-Kronecker for a fixed $\varepsilon$ [Graham and Hare 2008].

In this paper we transform the problem of determining the Kronecker constant $\kappa$ for a set of $d$ integers into a geometric problem in $\mathbb{R}^{d-1}$ which is equivalent to an optimization problem in convex analysis and prove that $\kappa$ can be computed in a finite number of steps. Using our geometric approach we have been able to show that the 'angular' Kronecker constant is always rational and deduce an (nontrivial) upper bound on the Kronecker constant for any given finite set. This gives improved estimates for the Kronecker constants of initial segments of geometric progressions and in some situations is sharp. We also explicitly determine the Kronecker constants for all two element sets.

For sets with three or more elements the problem of determining the Kronecker constant is inherently much more difficult and fundamentally different from the two element case. For instance, in [Graham and Hare 2006a] it was shown that for every pair of integers $a, b, \kappa\{a+n, b+n\} \rightarrow 0$ as $n \rightarrow \infty$. This is false if $\{a, b\}$ is replaced by any three element set. We calculate the exact Kronecker constant for various interesting classes of sets of size three. The answers can be surprisingly complicated. For example, for a sum set, $S=\{m, n, m+n\}, \kappa(S)$ depends on the $\bmod 3$ congruence of $m+2 n$; for an arithmetic progression of length three it depends on the step size $\bmod 4$. We also characterize the three element sets whose Kronecker constant is maximal.

The paper is organized as follows: In section two our formula is derived. The Kronecker constants for two element sets are determined in section three. In section four we consider geometric consequences of our approach, including proving that the angular Kronecker constant is always a rational number. We study the geometry problem specifically for $\mathbb{R}^{3}$ in section five. In section six the problem of calculating Kronecker constants for three element sets is investigated. Lastly, in section seven we briefly discuss a computer algorithm we wrote for calculating Kronecker constants. Examples of exact constants helped formulate theorems and motivated some of the proofs. Tables of some of the numerical results have been archived at [Hare and Ramsey 2011].

\section{The Kronecker Constant Formula}

2.1. Definitions. We begin by specializing our definitions to the case when the compact group $G$ is the circle group, which we identify with $[-1 / 2,1 / 2]$. Its dual is $\mathbb{Z}$. This will be our setting throughout the rest of the paper. 
Definition 1. A subset $S \subseteq \mathbb{Z}$ is called an $\varepsilon$-Kronecker set if for every function $f: S \rightarrow \mathbb{T}$ there exists $x \in[-1 / 2,1 / 2]$ such that

$$
|f(n)-\exp 2 \pi i n x|<\varepsilon \text { for all } n \in S .
$$

The Kronecker constant of $S$ is

$$
\kappa(S)=\inf \{\varepsilon: S \text { is } \varepsilon \text {-Kronecker }\} .
$$

As $|f(n)-\exp 2 \pi i n x| \leq 2$ for all integers $n$ and $x \in[-1 / 2,1 / 2]$, it follows that $\kappa(S) \leq 2$ for all non-empty sets $S$. This is sharp when $S$ contains 0 as can be seen by taking $f$ with $f(0)=-1$. As observed in [Graham and Hare 2006a], every finite subset of $\mathbb{Z} \backslash\{0\}$ is $\varepsilon$-Kronecker for some $\varepsilon<2$ and obviously $\kappa\{n\}=0$ for any $n \neq 0$. Thus for the rest of this paper we will assume $S$ is a subset of $\mathbb{Z} \backslash\{0\}$ with $d \geq 2$ elements.

Any complex number, $z$, of modulos 1 can be written uniquely as exp $2 \pi i \operatorname{Arg}(z)$ where $\operatorname{Arg}(z) \in[-1 / 2,1 / 2)$. Often it is convenient to measure differences in arguments and this leads to the following related constant. As usual, we write $l^{\infty}(S)$ for the set of bounded functions on $S$ with norm $\|f\|_{l^{\infty}(S)}=\sup \{|f(n)|: n \in S\}$.

Definition 2. For $f: S \rightarrow \mathbb{R}$, let

$$
\alpha_{S}(f)=\inf \left\{\|\operatorname{Arg}(\exp 2 \pi i(f(\cdot)-(\cdot) x))\|_{l_{\infty}(S)}: x \in[-1 / 2,1 / 2]\right\} .
$$

The angular Kronecker constant of $S$ is

$$
\alpha(S)=\sup \left\{\alpha_{S}(f) \mid f: S \rightarrow \mathbb{R}\right\} .
$$

One can easily see that $0 \leq \alpha(S) \leq 1 / 2$ and that $\kappa(S)=|\exp 2 \pi i \alpha(S)-1|$.

2.2. The $l^{\infty}$ distance in $\mathbb{R}^{d}$ from points to some lines. We begin with some elementary formulas for computing the quotient norms that will be helpful in deriving our formula for the Kronecker constant.

Given $S=\left\{n_{1}, \ldots, n_{d}\right\} \subseteq \mathbb{Z} \backslash\{0\}$, let $n=n(S)$ be the vector $\left(n_{1}, \ldots, n_{d}\right) \in \mathbb{R}^{d}$ and let $L(=L(S))$ be the line in $\mathbb{R}^{d}$ through the origin with direction vector $n$ :

$$
L=\{\lambda n: \lambda \in \mathbb{R}\} .
$$

For $f: S \rightarrow \mathbb{R}$ denote by $\|f\|_{L}$ the norm

$$
\|f\|_{L}=\inf _{x \in \mathbb{R}}\left(\max _{1 \leq j \leq d}\left|f\left(n_{j}\right)-n_{j} x\right|\right)=\inf _{x \in \mathbb{R}}\|f(\cdot)-(\cdot) x\|_{l^{\infty}(S)}
$$

Under the natural identification of $\mathbb{R}^{S}$ with $\mathbb{R}^{d},\|\cdot\|_{L}$ is the quotient norm on $\mathbb{R}^{d} / L$ induced by the $l^{\infty}(S)$ norm. Key to our approach is the observation that the angular Kronecker constant can be calculated in terms of this quotient norm.

Lemma 1. (i) For $f: S \rightarrow \mathbb{R}$,

$$
\alpha_{S}(f)=\inf \left\{\|f-k\|_{L}: k \in \mathbb{Z}^{d}\right\} .
$$

(ii) There exists a function $f$ such that $\alpha_{S}(f)=\alpha(S)$.

(iii) Given a non-zero integer $\lambda$, let $\lambda S=\{\lambda m: m \in S\}$. Then $\alpha(S)=\alpha(\lambda S)$.

Proof. (i) and (ii) are easy consequences of the periodicity of the exponential function and compactness. (iii) follows since the lines $\mathbb{R} n$ and $\mathbb{R} \lambda n$ coincide for non-zero $\lambda \in \mathbb{R}$. 
In particular, there is no loss in imposing the assumption that the greatest common divisor of the elements of $S$ is 1 .

Lemma 2. For every $u \in \mathbb{R}^{d}$ there is a scalar $s \in \mathbb{R}$ and integers $i<j$ such that

$$
\|u\|_{L}=\|u-s n\|_{l^{\infty}}=\left|s n_{i}-u_{i}\right|=\left|s n_{j}-u_{j}\right|=\frac{\left|n_{j} u_{i}-n_{i} u_{j}\right|}{\left|n_{i}\right|+\left|n_{j}\right|} .
$$

Furthermore, if $n_{i} n_{j}>0$, then $s n_{i}-u_{i}=-\left(s n_{j}-u_{j}\right)$, while if $n_{i} n_{j}<0$, then $s n_{i}-u_{i}=s n_{j}-u_{j}$.

Proof. By compactness, $\|u\|_{L}=\|u-s n\|_{l^{\infty}}$ for some $s \in \mathbb{R}$. We denote $\rho:=\|u\|_{L}$. If $\rho=0$, then $u=s n$ and for all $j \in\{1, \ldots, d\}$ we have $s n_{j}=u_{j}$. Thus $s n_{i}-u_{i}=$ $s n_{j}-u_{j}=-\left(s n_{j}-u_{j}\right)=0=\rho$ for all indices $i, j$. Trivially,

$$
\frac{\left|n_{j} u_{i}-n_{i} u_{j}\right|}{\left|n_{i}\right|+\left|n_{j}\right|}=0=\|u\|_{L} .
$$

So we can assume that $\rho>0$. Fix an index $i$ such that $\rho=\left|s n_{i}-u_{i}\right|$. If $\left|s n_{i}-u_{i}\right|>\left|s n_{j}-u_{j}\right|$ for all $j \neq i$, then for sufficiently small $\tau$, of a suitable choice of sign, $\left|s n_{i}-u_{i}\right|>\left|(s-\tau) n_{i}-u_{i}\right|>\left|(s-\tau) n_{j}-u_{j}\right|$ for all $j \neq i$. But then $\|u\|_{L} \leq\left|(s-\tau) n_{i}-u_{i}\right|<\rho$, giving a contradiction. Thus if we let $A$ be the set of all indices $j \in\{1, \ldots, d\}$ such that $\left|s n_{j}-u_{j}\right|=\rho$, then $A$ contains at least two indices. Put $B=\{1, \ldots, d\} \backslash A$.

For $j \in B$, set

$$
\delta_{j}:=\frac{\rho-\left|s n_{j}-u_{j}\right|}{2\left|n_{j}\right|}>0
$$

and for $j \in A$, set $\delta_{j}=\rho /\left|n_{j}\right|>0$. Then $\delta:=\min _{1 \leq j \leq d} \delta_{j}>0$. Put $\epsilon=$ $\operatorname{sign}\left(s n_{i}-u_{i}\right)$ and set $t=s-\epsilon \cdot \operatorname{sign}\left(n_{i}\right) \delta$ (where $i$ continues to denote the fixed index with $\left.\rho=\left|s n_{i}-u_{i}\right|\right)$.

Suppose that for every $j \in A$ either $n_{i} n_{j}>0$ and $s n_{i}-u_{i} \neq-\left(s n_{j}-u_{j}\right)$, or $n_{i} n_{j}<0$ and $s n_{i}-u_{i} \neq s n_{j}-u_{j}$. We will prove that in this case $\|t n-u\|_{\infty}<\rho$, which is a contradiction. To see this, first note that if $j \in B$, then

$$
\begin{aligned}
\left|t n_{j}-u_{j}\right| & =\left|s n_{j}-u_{j}-\varepsilon \cdot \operatorname{sign}\left(n_{i}\right) \delta n_{j}\right| \\
& \leq\left|s n_{j}-u_{j}\right|+\delta\left|n_{j}\right|=\frac{\rho+\left|s n_{j}-u_{j}\right|}{2}<\rho .
\end{aligned}
$$

If $j \in A$ and $n_{i} n_{j}>0$, then we must have $s n_{i}-u_{i}=s n_{j}-u_{j}($ even if $j=i)$. Thus

$$
t n_{j}-u_{j}=s n_{j}-u_{j}-\varepsilon \cdot \operatorname{sign}\left(n_{i}\right) \delta n_{j}=\varepsilon\left(\rho-\left|n_{j}\right| \delta\right) .
$$

As $0<\delta \leq \rho /\left|n_{j}\right|$, it follows that $\left|t n_{j}-u_{j}\right|=\rho-\left|n_{j} \delta\right|<\rho$. If $j \in A$ and $n_{i} n_{j}<0$, a similar argument gives the same conclusion. Consequently, $\|t n-u\|_{\infty}<\rho$ as claimed.

Finally, note that if $\left|s n_{i}-u_{i}\right|=\left|s n_{j}-u_{j}\right|, n_{i} n_{j}>0$ and $s n_{i}-u_{i}=-\left(s n_{j}-u_{j}\right)$, then $s=\frac{u_{i}+u_{j}}{n_{i}+n_{j}}$. Hence $\rho=\left|s n_{i}-u_{i}\right|=\frac{\left|n_{j} u_{i}-n_{i} u_{j}\right|}{\left|n_{i}\right|+n_{j} \mid}$ and so the fractional formula holds. The case $n_{i} n_{j}<0$ is similar.

Proposition 1. For all $u \in \mathbb{R}^{d}$,

$$
\|u\|_{L}=\max \left\{\frac{\left|n_{j} u_{i}-n_{i} u_{j}\right|}{\left|n_{i}\right|+\left|n_{j}\right|} \quad: \quad 1 \leq i<j \leq d\right\}
$$


Proof. We proceed by duality, noting that if $W=\mathbb{R}^{d} / L$ then $W^{*}=L^{\perp}$, where $L^{\perp}$ consists of all $g \in \mathbb{R}^{d}$ such that $g(L)=\{0\}$. The quotient norm of $u+L$ satisfies

$$
\|u\|_{L}=\|u+L\|_{W}=\sup \left\{\frac{|g(u)|}{\|g\|_{1}} \quad: \quad g \in L^{\perp} \backslash\{0\}\right\} .
$$

Fix $1 \leq i<j \leq d$. Let $e$ satisfy $e_{k}=0$ for $k \notin\{i, j\}$, but $e_{i}=n_{j}$ and $e_{j}=-n_{i}$. Then $e \in L^{\perp}$ and $\|e\|_{1}=\left|n_{i}\right|+\left|n_{j}\right|>0$. Consequently,

$$
\|u+L\|_{W} \geq \frac{|e(u)|}{\|e\|_{1}}=\frac{\left|n_{j} u_{i}-n_{i} u_{i}\right|}{\left|n_{i}\right|+\left|n_{j}\right|}
$$

By Lemma 2 there are $i \neq j$ in $\{1, . ., d\}$ such that $\|u\|_{L}=\frac{\left|n_{j} u_{i}-n_{i} u_{j}\right|}{\left|n_{i}\right|+\left|n_{j}\right|}$. The desired equality now follows.

Remark 1. Proposition 1 specifies a set of $d(d-1)$ linear functionals with which one can compute $\|u\|_{L}$. This is a minimal set in the following sense: For each of the linear functionals, say $L_{i, j}(u)=\left(n_{j} u_{i}-n_{i} u_{j}\right) /\left(\left|n_{i}\right|+\left|n_{j}\right|\right)$ with $1 \leq i<j \leq d$, there is some $u$ such that for all $1 \leq r<s \leq d$ with $(r, s) \neq(i, j)$,

$$
\|u\|_{L}=\left|L_{i, j}(u)\right|>\left|L_{r, s}(u)\right| \text {. }
$$

To see this, let $u$ be the vector with $u_{i}=\operatorname{sign}\left(n_{j}\right), u_{j}=-\operatorname{sign}\left(n_{i}\right)$ and all other coordinates equal to 0 . Then

$$
L_{i, j}(u)=\frac{n_{j} \operatorname{sign}\left(n_{j}\right)-n_{i}\left(-\operatorname{sign}\left(n_{i}\right)\right)}{\left|n_{i}\right|+\left|n_{j}\right|}=1 .
$$

If $\{r, s\} \cap\{i, j\}=\emptyset$, then $L_{r, s}(u)=0$. Otherwise if, say, $i \in\{r, s\}$ and $t \in$ $\{r, s\} \backslash\{i, j\}$, then

$$
\left|L_{r, s}(u)\right|=\frac{\left|n_{i} u_{i}\right|}{\left|n_{i}\right|+\left|n_{t}\right|}=\frac{\left|n_{i}\right|}{\left|n_{i}\right|+\left|n_{t}\right|}<1 .
$$

2.3. Dimensional and Symmetry Reductions for Computing $\alpha$. Given a finite set $S$ and line $L=L(S)$, as described before, we let $\mathcal{L}$ be the subgroup of $\mathbb{R}^{d}$ generated by $L$ and $\mathbb{Z}^{d}$, and put

$$
\mathcal{K}=\mathcal{L} \cap\left(\mathbb{R}^{d-1} \times\{0\}\right) .
$$

We will simplify the calculation of $\alpha_{S}(f)=\inf \left\{\|f-k\|_{L}: k \in \mathbb{Z}^{d}\right\}$ and $\alpha(S)=$ $\sup \left\{\alpha_{S}(f) \mid f: S \rightarrow \mathbb{R}\right\}$ by reducing the search space for $f$ by one dimension and showing that we may replace $k \in \mathbb{Z}^{d}$ by $k \in \mathcal{K}$.

We first highlight the following useful information about $\mathcal{K}$.

Lemma 3. $k \in \mathcal{K}$ if and only if $k_{d}=0$ and $k=\left(s / n_{d}\right) n+q$ for some $s \in \mathbb{Z}$ and $q \in \mathbb{Z}^{d}$. Equivalently, $k \in \mathcal{K}$ if and only if $k_{d}=0$ and there is some integer $s$ with

$$
k \bmod 1=\left(s / n_{d}\right) n \bmod 1 .
$$

Moreover, $\mathcal{K}$ has only finitely many points in each bounded subset of $\mathbb{R}^{d-1} \times\{0\}$.

Remark 2. In particular, $\mathcal{K}$ is a subgroup of $\mathbb{Q}^{d-1} \times\{0\}$.

Proof. The equivalence of the two descriptions is obvious, as is sufficiency. To see the first, note that as $\mathcal{K} \subseteq \mathcal{L}$ we have $k=t n+q$ for some $t \in \mathbb{R}$ and $q \in \mathbb{Z}^{d}$. Since $k_{d}=0$, we must have $t=-q_{d} / n_{d}$, which gives the required form with $s=-q_{d} \in \mathbb{Z}$.

There are only finitely many points of $\mathcal{K}$ in any bounded set since $\mathcal{K}$ is a subset of the discrete lattice $\mathbb{Z}^{d} / n_{d}$. 
Here is the promised reduction for calculating $\alpha(S)$ and $\alpha_{S}(f)$.

Proposition 2. (i) $\alpha(S)=\sup \left\{\alpha_{S}(f): f \in \mathbb{R}^{d-1} \times\{0\}\right\}$.

(ii) For $f \in \mathbb{R}^{d-1} \times\{0\}, \alpha_{S}(f)=\inf \left\{\|f-k\|_{L}: k \in \mathcal{K}\right\}$.

(iii) For $f \in \mathbb{R}^{d-1} \times\{0\}$ and $h \in \mathcal{K}, \alpha_{S}(f)=\alpha_{S}(f-h)$ and $\alpha_{S}(f)=\alpha_{S}(-f)$.

Proof. (i) If $g \in \mathbb{R}^{d}$, then $f=g-\left(g_{d} / n_{d}\right) n \in \mathbb{R}^{d-1} \times\{0\}$. Because $\left(g_{d} / n_{d}\right) n \in L$, we have $(g-k)-L=(f-k)-L$ for all $k \in \mathbb{Z}^{d}$. Therefore, $\alpha_{S}(g)=\alpha_{S}(f)$. It follows that

$$
\alpha(S)=\sup \left\{\alpha_{S}(g): g \in \mathbb{R}^{d}\right\} \leq \sup \left\{\alpha_{S}(f): f \in \mathbb{R}^{d-1} \times\{0\}\right\}
$$

The reverse inequality is immediate.

(ii) Let $f \in \mathbb{R}^{d-1} \times\{0\}$ and suppose $k \in \mathbb{Z}^{d}$. Let $x=-k_{d} / n_{d}$. Then the $d$-th coordinate of $h=k+x n$ is 0 , thus $h \in \mathcal{K}$. Because $h-k=x n \in L$, we have $(f-k)-L=(f-h)-L$. It follows that $\|f-k\|_{L}=\|f-h\|_{L}$ and therefore

$$
\alpha_{S}(f)=\inf \left\{\|f-k\|_{L}: k \in \mathbb{Z}^{d}\right\} \geq \inf \left\{\|f-h\|_{L}: h \in \mathcal{K}\right\}
$$

To prove the reversed inequality, consider any $k \in \mathcal{K}$. Then $k$ has the form $x n+q$ for some $x \in \mathbb{R}$ and $q \in \mathbb{Z}^{d}$. Because $x n \in L$, we have $(f-k)-L=$ $f-(x n+q)-L=(f-q)-L$. It follows that $\|f-k\|_{L}=\|f-q\|_{L}$ and therefore

$$
\inf \left\{\|f-k\|_{L}: k \in \mathcal{K}\right\} \geq \inf \left\{\|f-k\|_{L}: k \in \mathbb{Z}^{d}\right\}=\alpha_{S}(f) .
$$

(iii) holds because $\mathcal{K}$ is a group.

2.4. Group Generators. Next, we offer more detailed descriptions of the set $\mathcal{K}$ by identifying sets of linearly independent group generators.

Proposition 3. Suppose that vectors $\left\{P^{(j)}\right\}_{j=1}^{d-1} \subseteq \mathbb{R}^{d}$ satisfy the following three properties:

(i) For each $1 \leq j \leq d-1$ there is some integer s such that $P^{(j)}-s n / n_{d} \in \mathbb{Z}^{d}$;

(ii) $P_{i}^{(j)}=0$ for $j<i \leq d$;

(iii) $P_{j}^{(j)}=\frac{\operatorname{gcd}\left(n_{j}, \ldots, n_{d}\right)}{\operatorname{gcd}\left(n_{j+1}, \ldots, n_{d}\right)}$ for $1 \leq j \leq d-1$.

Then $\left\{P^{(j)}\right\}_{j=1}^{d-1}$ is a vector space basis for $\mathbb{R}^{d-1} \times\{0\}$ and a generating set for $\mathcal{K}$ as a group.

Here $P_{i}^{(j)}$ denotes coordinate $i$ of vector $P^{(j)}$.

Remark 3. Note that the first property implies that $P^{(j)} \in \mathbb{Z}^{d} / n_{d}$ and is equivalent to the statement that $P^{(j)} \equiv$ sn mod 1 with modularity computed independently in each coordinate.

Proof. By the upper triangular structure of the $d \times(d-1)$ matrix whose $j$-th column is $P^{(j)}$, with positive elements on the diagonal, these vectors are linearly independent over $\mathbb{R}$ and therefore generate $\mathbb{R}^{d-1} \times\{0\}$.

As $P_{d}^{(j)}=0$ and $P^{(j)}-s n / n^{d} \in \mathbb{Z}^{d}$, each $P^{(j)} \in \mathcal{K}$. Suppose that $S=\left\{P^{(j)}\right\}_{j=1}^{d-1}$ does not generate $\mathcal{K}$ as a group. Then there is some $R \in \mathcal{K}$ outside the subgroup generated by $S$. There are unique real numbers $\alpha_{j}$ such that $R=\sum_{j=1}^{d-1} \alpha_{j} P^{(j)}$. Let $\beta_{j}=\alpha_{j}-\left\lfloor\alpha_{j}\right\rfloor$ and set $W=\sum_{j=1}^{d-1}\left\lfloor\alpha_{j}\right\rfloor P^{(j)}$. Because $\mathcal{K}$ is group we have $W \in \mathcal{K}$ and thus $R-W \in \mathcal{K}$. Hence there is some integer $r$ and vector $q \in \mathbb{Z}^{d}$ such that $R-W=\left(r / n_{d}\right) n+q$. 
Clearly, there is some $j$ such that $\beta_{j}>0$; let $J$ denote the largest such integer $j$. Then $R-W=\sum_{j=0}^{J} \beta_{j} P^{(j)}$. For $i>J$ and $j \leq J$, we have $P_{i}^{(j)}=0$ and therefore

$$
0=(R-W)_{i}=\left(r / n_{d}\right) n_{i}+q_{i}
$$

Thus $n_{d} / \operatorname{gcd}\left(n_{i}, n_{d}\right)$ divides $r$ for $i>J$. It follows that $n_{d} / \operatorname{gcd}\left(n_{J+1}, \ldots, n_{d}\right)$ divides $r$.

As $P_{J}^{(j)}=0$ for $j<J$, we have $(R-W)_{J}=\beta_{J} P_{J}^{(J)}$. Because $0<\beta_{J}<1$, we have

$$
0<(R-W)_{J}=\left(r / n_{d}\right) n_{J}+q_{J}<P_{J}^{(J)}
$$

By the previous paragraph, there is some integer $t$ such that $r=t n_{d} / w$ where $w=\operatorname{gcd}\left(n_{J+1}, \ldots, n_{d}\right)$. Thus

$$
(R-W)_{J}=t n_{J} / w+q_{J}=\frac{t n_{J}+q_{J} w}{w}
$$

Note that the $\operatorname{gcd}\left(n_{J}, \ldots, n_{d}\right)$ divides both $n_{J}$ and $w$. Therefore $(R-W)_{J}$ is an integer multiple of $\operatorname{gcd}\left(n_{J}, \ldots, n_{d}\right) / w=P_{J}^{(J)}$. This contradicts having $0<$ $(R-W)_{J}<P_{J}^{(J)}$.

Example 1. Consider $S=\left\{1, m, m^{2}, \ldots, m^{d-1}\right\} \subseteq \mathbb{Z}$, where $m>1$, and set $n=\left(1, m, \ldots, m^{d-1}\right)$. We can let $P^{(j)}=\left(m^{d-j} / m^{d-1}\right) n \bmod 1=m^{-j+1} n \bmod 1$ for $1 \leq j \leq d-1$. Then

$$
P_{s}^{(j)}=\left\{\begin{array}{ll}
m^{s-j-1} & \text { for } s \leq j \\
0 & \text { for } s>j
\end{array} .\right.
$$

The next result asserts that there are indeed bases as described in the previous result.

Proposition 4. For $1 \leq j \leq d-1$, there are vectors $P^{(j)} \in \mathcal{K}$ such that $P_{s}^{(j)}=0$ for $j<s \leq d ; P_{s}^{(j)} \in[0,1)$ for $s<j$; and

$$
\begin{aligned}
P_{j}^{(j)} & =\min \left\{q_{j}: q \in \mathcal{K}, q_{s} \in[0,1) \text { for } s<j, q_{j}>0, q_{s}=0 \text { for } s>j\right\} \\
& =\frac{\operatorname{gcd}\left(n_{j}, \ldots, n_{d}\right)}{\operatorname{gcd}\left(n_{j+1}, \ldots, n_{d}\right) .}
\end{aligned}
$$

Moreover,

$$
\left\{q \in \mathcal{K}: q_{s} \in[0,1) \text { for all } s\right\}=\left\{\left(t / n_{d}\right) n \bmod 1: 0 \leq t<n_{d-1}\right\}
$$

Proof. Let $1 \leq j \leq d-1$. If $e(j)$ is the canonical $d$-vector that has zero coordinates except the $j$-th, which is 1 , then for $j<d$ we have $e(j) \in \mathbb{Z}^{d-1} \times\{0\} \subset \mathcal{K}$. Hence the set

$$
S_{j}=\left\{q \in \mathcal{K}: q_{s} \in[0,1) \text { for } s<j, q_{j}>0, q_{s}=0 \text { for } s>j\right\}
$$

is not empty.

Suppose $r=\operatorname{gcd}\left(n_{j+1}, \ldots, n_{d}\right)$ and $r^{\prime}=\operatorname{gcd}\left(n_{j}, \ldots, n_{d}\right)$. Since $r^{\prime}=\operatorname{gcd}\left(n_{j}, r\right)$, there are integers $u$ and $v$ such that $r^{\prime}=u n_{j}+r v$. Consider $q=(u / r) n+k$ where $k \in \mathbb{Z}^{d}$ is to be specified. Note that $q \in \mathcal{L}$ for any such choice for $k$.

For $s>j$, choose $k_{s}=-u n_{s} / r$ so that $q_{s}=0$. Because $r$ divides $n_{s}, k_{s}$ is an integer. Because $q_{d}=0$, this puts $q \in \mathcal{K}$. For $s=j$, let $k_{j}=v$. Then $q_{j}=(u / r) n_{j}+v=\frac{u n_{j}+r v}{r}=\frac{r^{\prime}}{r}$. For $s<j$ choose $k_{s}$ to be an integer such that $(u / r) n_{s}+k_{s} \in[0,1)$. 
Consequently, $q \in S_{j}$ and we have proved that $\frac{r^{\prime}}{r} \in\left\{q_{j}: q \in S_{j}\right\}$.

Now consider any $q \in S_{j}$. Because $q \in \mathcal{K}$, by Lemma 3 there is an integer $m$ and $k \in \mathbb{Z}^{d}$ such that $q=\left(m / n_{d}\right) n+k$. Let $m / n_{d}=a / b$ with $\operatorname{gcd}(a, b)=1$. Since $q_{s}=0$ for $s>j$, we have $(a / b) n_{s}+k_{s}=0$. It follows that $a \mid k_{s}$ and $b \mid n_{s}$ for each $s>j$. Consequently, $b \mid \operatorname{gcd}\left(n_{j+1}, \ldots, n_{d}\right)$. So we may write $a / b$ as $c / r$, for some integer $c$. Then

$$
q_{j}=(c / r) n_{j}+k_{j}=\frac{c n_{j}+r k_{j}}{r}
$$

Since $r^{\prime} \mid r$ and $r^{\prime} \mid n_{j}$, we know that $q_{j}$ is an integer multiple of $r^{\prime} / r$ and because $q_{j}>0$, we have $q_{j} \geq r^{\prime} / r$.

Therefore $\min \left\{q_{j}: q \in S_{j}\right\}=r^{\prime} / r$ and hence for each $j$ there is at least one $P^{(j)}$ as specified in the lemma.

We turn to the last claim. Let $q=\left(t / n_{d}\right) n \bmod 1$ for some integer $t$. There is an vector $v \in \mathbb{Z}^{d}$ such that $q=\left(t / n_{d}\right) n-v$. Thus $q \in \mathcal{L}$. Because the $d$-th coordinate of $\left(t / n_{d}\right) n$ is an integer, we have $q_{d}=0$ and thus $q \in \mathcal{K}$. Conversely, suppose $q \in \mathcal{K}$ with $q_{s} \in[0,1)$ for all $s$. By Lemma 3 there is some integer $t$ and vector $v \in \mathbb{Z}^{d}$ such that $q=\left(t / n_{d}\right) n+v$. There is some integer $t^{\prime} \in\left[0, n_{d}\right)$ such that $t=t^{\prime}+w n_{d}$ for some integer $w$. Because $\left(w n_{d} / n_{d}\right) n$ is an integer vector, we have

$$
\left(t^{\prime} / n_{d}\right) n \bmod 1=\left(t / n_{d}\right) n \bmod 1=\left(\left(t / n_{d}\right) n+v\right) \bmod 1=q \bmod 1=q .
$$

Corollary 1. The lattice $\mathcal{K}$ is generated by vectors which span a $d$-1-dimensional parallelotope of volume $\operatorname{gcd}\left(n_{1}, \ldots, n_{d}\right) / n_{d}$.

Proof. The volume of the parallelotope generated by the vectors $P^{(j)}, j=1, \ldots, d-1$ is equal to $\left|\operatorname{det}\left[P^{(j)}\right]\right|$ where here we think of $P^{(j)}$ as vectors in $\mathbb{R}^{d-1}$, excluding the last (zero) coordinate. The form of these vectors makes it easy to see that this determinant is $\operatorname{gcd}\left(n_{1}, \ldots, n_{d}\right) / n_{d}$.

Remark 4. Propositions 3 and 4 allows an algorithm for choosing $P^{(j)}$ 's. Let

$$
W=\left\{\left(k / n_{d}\right) n \bmod 1: 0 \leq k<n_{d}-1\right\}
$$

If Prop. 3 specifies that $P_{j}^{(j)}<1$, we search $W$ for $P^{(j)}$ with the minimization conditions specified in Prop. 4. If Prop. 3 specifies $P_{j}^{(j)}=1$, we use the canonical basis vector $e(j)$ (which is in $\mathcal{K}$ because $j<d$ ).

Example 2. Let $S \subset \mathbb{Z} \backslash\{0\}$ have d elements, two of which are relatively prime. Enumerate $S$ as $\left\{n_{1}, \ldots, n_{d}\right\}$ with $\operatorname{gcd}\left(n_{d-1}, n_{d}\right)=1$. Let $\left\{P^{(j)}\right\}_{j=1}^{d-1}$ be a generating set for $\mathcal{K}$ as described in Prop. 4. Here $P_{d-1}^{(d-1)}=1 / n_{d}$, while $P_{j}^{(j)}=1$ for $j<d-1$. By Prop. 3, we may substitute the canonical basis vector $(j)$ for $P^{(j)}$ when $j<d-1$ and still have a generating set for $\mathcal{K}$.

2.5. Our Main Result. We continue to let $\mathcal{L}$ be the subgroup of $\mathbb{R}^{d}$ generated by the line $\mathbb{R} n$ and $\mathbb{Z}^{d}$ and set $\mathcal{K}=\mathcal{L} \cap\left(\mathbb{R}^{d-1} \times\{0\}\right)$. We can calculate the Kronecker constant in terms of $\mathcal{K}$.

Theorem 1. Suppose $S=\left\{n_{1}, n_{2}, \ldots, n_{d}\right\} \subseteq \mathbb{Z} \backslash\{0\}$ and assume $P^{(1)}, \ldots, P^{(d-1)} \in$ $\mathbb{R}^{d}$ are generators of the associated group $\mathcal{K}$. The angular Kronecker constant is 
the smallest number $E$ such that for all $\left(x_{1}, \ldots, x_{d}\right) \in \mathbb{R}^{d-1} \times\{0\}$ there are integers $r_{1}, \ldots, r_{d-1}$ such that

$$
\max \left\{\frac{\left|n_{j}\left(x_{i}-\sum_{m=1}^{d-1} r_{m} P_{i}^{(m)}\right)-n_{i}\left(x_{j}-\sum_{m=1}^{d-1} r_{m} P_{j}^{(m)}\right)\right|}{\left|n_{i}\right|+\left|n_{j}\right|}: 1 \leq i<j \leq d\right\} \leq E .
$$

Proof. According to Proposition 2 we can calculate $\alpha(S)$ with $f=\left(x_{1}, x_{2}, \ldots, x_{d-1}, 0\right) \in$ $\mathbb{R}^{d-1} \times\{0\}$ and elements of $\mathcal{K}$ :

$$
\alpha(S)=\sup \left\{\inf _{k \in \mathcal{K}}\|f-k\|_{L}: f \in \mathbb{R}^{d-1} \times\{0\}\right\} .
$$

By Proposition 1,

$$
\|f-k\|_{L}=\max \left\{\frac{\left|n_{j}(f-k)_{i}-n_{i}(f-k)_{j}\right|}{\left|n_{i}\right|+\left|n_{j}\right|}: 1 \leq i<j \leq d\right\}
$$

and therefore

$$
\alpha(S)=\sup _{\left(x_{1}, \ldots, x_{d}\right) \in \mathbb{R}^{d-1} \times\{0\}}\left(\inf _{k \in \mathcal{K}}\left[\max _{i \neq j}\left\{\frac{\left|n_{j}\left(x_{i}-k_{i}\right)-n_{i}\left(x_{j}-k_{j}\right)\right|}{\left|n_{i}\right|+\left|n_{j}\right|}\right\}\right]\right) .
$$

If $P^{(1)}, \ldots, P^{(d-1)}$ generate $\mathcal{K}$ as a group, then each $k \in \mathcal{K}$ can be written as $\sum_{m=1}^{d-1} r_{m} P^{(m)}$ for integers $r_{1}, \ldots, r_{d-1}$. Thus $\alpha_{S}(f)$ equals

$$
\inf _{r_{1}, \ldots, r_{d-1} \in \mathbb{R}}\left(\max _{1 \leq i<j \leq d}\left\{\frac{\left|n_{j}\left(x_{i}-\sum_{m=1}^{d-1} r_{m} P_{i}^{(m)}\right)-n_{i}\left(x_{j}-\sum_{m=1}^{d-1} r_{m} P_{j}^{(m)}\right)\right|}{\left|n_{i}\right|+\left|n_{j}\right|}\right\}\right) .
$$

The statement in the theorem follows directly.

Remark 5. When $k \in \mathcal{K}, k_{d}=0$. Thus $\alpha(S)$ is the smallest number $E$ such that for all $\left(x_{1}, \ldots, x_{d-1}\right) \in \mathbb{R}^{d-1}$ there are integers $r_{1}, \ldots, r_{d-1}$ such that

$$
\frac{\left|n_{j}\left(x_{i}-\sum_{m=1}^{d-1} r_{m} P_{i}^{(m)}\right)-n_{i}\left(x_{j}-\sum_{m=1}^{d-1} r_{m} P_{j}^{(m)}\right)\right|}{\left|n_{i}\right|+\left|n_{j}\right|} \leq E \text { for } 1 \leq i<j \leq d-1
$$

and

$$
\frac{\left.\mid n_{d}\left(x_{t}-\sum_{m=1}^{d-1} r_{m} P_{t}^{(m)}\right)\right) \mid}{\left|n_{t}\right|+\left|n_{d}\right|} \leq E \text { for } 1 \leq t \leq d-1 .
$$

\section{KRONECKER CONSTANTS OF TWO ELEMENT SETS}

3.1. Formula for two element sets. When $d=2$, the formula derived in the previous section is very simple and we can completely determine the Kronecker constants.

Proposition 5. For distinct, non-zero integers $n_{1}$ and $n_{2}$,

$$
\alpha\left\{n_{1}, n_{2}\right\}=\sup _{x \in \mathbb{R}}\left(\inf _{r \in \mathbb{Z}} \frac{\left|n_{2}\left(x-r \cdot \operatorname{gcd}\left(n_{1}, n_{2}\right) / n_{2}\right)\right|}{\left|n_{1}\right|+\left|n_{2}\right|}\right)=\frac{\operatorname{gcd}\left(n_{1}, n_{2}\right)}{2\left(\left|n_{1}\right|+\left|n_{2}\right|\right)}
$$

Proof. When $d=2, \mathcal{K}$ is generated by the single vector $P^{(1)}=\operatorname{gcd}\left(n_{1}, n_{2}\right) / n_{2}{ }^{2}$ (see Prop. 4) so the first equality follows directly from Theorem 1 or Remark 4.

\footnotetext{
${ }^{2}$ We typically view the vectors $P^{(j)}$ as belonging to $\mathbb{R}^{d-1}$, suppressing the final (zero) coordinate.
} 
Temporarily fix $x$ and choose an integer $r$ such that $\left|x-r \operatorname{gcd}\left(n_{1}, n_{2}\right) / n_{2}\right| \leq$ $\operatorname{gcd}\left(n_{1}, n_{2}\right) /\left(2\left|n_{2}\right|\right)$. Then

$$
\frac{\left|n_{2}\left(x-r \operatorname{gcd}\left(n_{1}, n_{2}\right) / n_{2}\right)\right|}{\left|n_{1}\right|+\left|n_{2}\right|} \leq \frac{\operatorname{gcd}\left(n_{1}, n_{2}\right)}{2\left(\left|n_{1}\right|+\left|n_{2}\right|\right)}
$$

and hence $\alpha\left\{n_{1}, n_{2}\right\} \leq \operatorname{gcd}\left(n_{1}, n_{2}\right) / 2\left(\left|n_{1}\right|+\left|n_{2}\right|\right)$. To verify the second equality, just take $x=\operatorname{gcd}\left(n_{1}, n_{2}\right) /\left(2 n_{2}\right)$.

Example 3. $\alpha\{-1,1\}=1 / 4$, and hence $\kappa\{-1,1\}=\sqrt{2}$. In fact, it is clear from the formula above that $\alpha\left\{n_{1}, n_{2}\right\} \leq 1 / 4$ for all $n_{1}, n_{2}$ and therefore $\kappa\left\{n_{1}, n_{2}\right\} \leq \sqrt{2}$. Equality occurs if and only if $n_{1}=-n_{2}$.

The following are some easy corollaries.

Corollary 2. Given distinct non-zero integers $n_{i}, 1 \leq i \leq d$,

$$
\alpha\left\{n_{1}, \ldots, n_{d}\right\} \geq \sup \left\{\frac{\operatorname{gcd}\left(n_{i}, n_{j}\right)}{2\left|n_{i}\right|+2\left|n_{j}\right|}: 1 \leq i<j \leq d\right\}
$$

Proof. The Kronecker constant is clearly non-decreasing with respect to the subset relation.

Corollary 3. (i) For all positive integers $n, m, j$

$$
\alpha\{n, m n+j\}<\alpha\{n, m n\}=\frac{1}{2(m+1)} .
$$

(ii) For any distinct integers $n_{1}, n_{2}, \lim _{m \rightarrow \infty} \alpha\left\{n_{1}+m, n_{2}+m\right\}=0$.

(iii) If $n_{1} \neq 0$, then $\lim _{m \rightarrow \infty} \alpha\left\{n_{1}, n_{2}+m\right\}=0$.

Proof. (i) Indeed, if $p=\operatorname{gcd}(n, m n+j)$ with $n=p N$ and $j=p J$, then

$$
\alpha\{n, m n+j\}=\frac{1}{2(N+N m+J)}<\frac{1}{2(m+1)} .
$$

(ii) If not, then for some $\alpha_{0}>0$ and subsequence $m_{k} \rightarrow \infty, \alpha\left\{n_{1}+m_{k}, n_{2}+m_{k}\right\} \geq$ $\alpha_{0}$. Hence if $p=\operatorname{gcd}\left(n_{1}+m_{k}, n_{2}+m_{k}\right)$ then $p \geq \alpha_{0} 2\left(n_{1}+n_{2}+2 m_{k}\right)$. But $p$ must divide $n_{2}-n_{1}$, so this is impossible.

(iii) This is similar as the $\operatorname{gcd}\left(n_{1}, n_{2}+m_{k}\right)$ is bounded by $n_{1}$.

Alternate proofs of this corollary can be found in [Graham and Hare 2006a].

Remark 6. The Kronecker constant does not decay monotonically in either (ii) or (iii). For example, $\alpha\{1,3\}=1 / 8$, while $\alpha\{1+1,3+1\}=1 / 6$. Similarly, $\alpha\{2,3\}=1 / 10<\alpha\{2,4\}$.

3.2. Asymptotic results for translates of three element sets. The analogue of Cor. 3(ii) fails for every three element set. Indeed, it is easy to prove the following facts.

Proposition 6. Suppose that $n_{1}<n_{2}<n_{3}$.

(i) For any integer $m$,

$$
\alpha\left\{n_{1}+m, n_{2}+m, n_{3}+m\right\} \geq \frac{1}{2} \max _{i \neq j, k}\left(\alpha\left\{n_{j}-n_{i}, n_{k}-n_{i}\right\}\right) .
$$

(ii) If $n_{2}-n_{1}, n_{3}-n_{1}, n_{3}-n_{2}$ are pairwise coprime, then

$$
\limsup _{m \rightarrow \infty} \alpha\left\{n_{1}+m, n_{2}+m, n_{3}+m\right\}=\frac{1}{4\left(n_{3}-n_{1}\right)} .
$$


(iii) $\lim \sup _{m \rightarrow \infty} \alpha\left\{n_{1}, n_{2}, m\right\}=\alpha\left\{n_{1}, n_{2}\right\}$.

Proof. To keep the notation simpler we will write $A(z)$ for $\operatorname{Arg}(\exp 2 \pi i z)$.

(i) Let $\alpha_{m}=\alpha\left\{n_{1}+m, n_{2}+m, n_{3}+m\right\}$. Let $f:\left\{n_{2}-n_{1}, n_{3}-n_{1}\right\} \rightarrow \mathbb{R}$ be given and define $g$ by $g\left(n_{1}+m\right)=0$ and $g\left(n_{j}+m\right)=f\left(n_{j}-n_{1}\right)$ for $j=2,3$. We may choose $x$ such that $|A(g(n)-n x)| \leq \alpha_{m}$ for $n=n_{j}+m, j=1,2,3$.

By the triangle inequality,

$$
\begin{aligned}
& \left|A\left(f\left(n_{2}-n_{1}\right)-x\left(n_{2}-n_{1}\right)\right)\right| \leq\left|A\left(f\left(n_{2}-n_{1}\right)-x\left(n_{2}+m\right)\right)\right|+\left|A\left(x\left(m+n_{1}\right)\right)\right| \\
& \quad=\left|A\left(g\left(n_{2}+m\right)-x\left(n_{2}+m\right)\right)\right|+\left|A\left(x\left(m+n_{1}\right)-g\left(n_{1}+m\right)\right)\right| \leq 2 \alpha_{m} .
\end{aligned}
$$

Similarly,

$$
\left|A\left(f\left(n_{3}-n_{1}\right)-x\left(n_{3}-n_{1}\right)\right)\right| \leq 2 \alpha_{m}
$$

This proves $2 \alpha_{m} \geq \alpha\left\{n_{2}-n_{1}, n_{3}-n_{1}\right\}$. This argument remains valid under permutations of $n_{1}, n_{2}$ and $n_{3}$.

(ii) Let $f:\left\{n_{1}+m, n_{2}+m, n_{3}+m\right\} \rightarrow \mathbb{R}$ and for notational convenience put $\theta_{j}=f\left(n_{j}+m\right)$. The two-element formula for coprime sets implies that

$$
\begin{aligned}
& \alpha\left\{n_{2}-n_{1}, n_{3}-n_{1}\right\}=\frac{1}{2\left(n_{3}+n_{2}-2 n_{1}\right)} \equiv \alpha_{1} \\
& \alpha\left\{n_{1}-n_{2}, n_{3}-n_{2}\right\}=\frac{1}{2\left(n_{3}-n_{1}\right)} \equiv \alpha_{2} \\
& \alpha\left\{n_{1}-n_{3}, n_{2}-n_{3}\right\}=\frac{1}{2\left(2 n_{3}-n_{2}-n_{1}\right)} \equiv \alpha_{3} .
\end{aligned}
$$

Choose angles $x_{1}, x_{2}, x_{3}$ such that for each $l=1,2,3$,

$$
\left|A\left(\left(n_{j}-n_{l}\right) x_{l}-\left(\theta_{j}-\theta_{l}\right)\right)\right| \leq \alpha_{l} \text { for } j \in\{1,2,3\}, j \neq l .
$$

Fix $\varepsilon>0$ and choose intervals $I_{l}$, containing $x_{l}$, such that if $y_{l} \in I_{l}$, then $\left|n_{j}\left(x_{l}-y_{l}\right)\right| \leq \varepsilon$. Take any $m$ so that $m \cdot \min \left\{\left|I_{l}\right|: 1 \leq l \leq 3\right\}>1$. These intervals contain a full period of the function $\exp 2 \pi i m(\cdot)$ and thus there exists $y_{l} \in I_{l}$ such that $\exp 2 \pi i m y_{l}=\exp 2 \pi i\left(\theta_{l}-n_{l} x_{l}\right)$. It follows that

$$
\left|A\left(\left(n_{l}+m\right) y_{l}-\theta_{l}\right)\right|=\left|A\left(n_{l}\left(y_{l}-x_{l}\right)\right)\right| \leq \varepsilon,
$$

while for $j \neq l$,

$$
\begin{aligned}
\left|A\left(\left(n_{j}+m\right) y_{l}-\theta_{j}\right)\right| & \leq\left|A\left(n_{j}\left(y_{l}-x_{l}\right)\right)\right|+\left|A\left(\left(n_{j}-n_{l}\right) x_{l}-\left(\theta_{j}-\theta_{l}\right)\right)\right| \\
& \leq \varepsilon+\alpha_{l} .
\end{aligned}
$$

Put

$$
t_{1}=\frac{n_{3}+n_{2}-2 n_{1}}{4\left(n_{3}-n_{1}\right)}, t_{2}=\frac{1}{4}, t_{3}=\frac{2 n_{3}-\left(n_{2}+n_{1}\right)}{4\left(n_{3}-n_{1}\right)}
$$

and let $y=t_{1} y_{1}+t_{2} y_{2}+t_{3} y_{3}$. As $y$ is a convex combination of $y_{1}, y_{2}, y_{3}$ we have

$$
\left|A\left(\left(n_{l}+m\right) y-\theta_{l}\right)\right| \leq t_{l} \varepsilon+t_{j}\left(\alpha_{j}+\varepsilon\right)+t_{k}\left(\alpha_{k}+\varepsilon\right)
$$

where $j, k \neq l$. Each of these sums is $1 / 4\left(n_{3}-n_{1}\right)+\varepsilon$ and as $\varepsilon>0$ is arbitrary, this completes the proof.

(iii) is similar, but easier.

Remark 7. Of course, a similar argument can be given in (ii) if the integers $n_{2}-n_{1}, n_{3}-n_{1}, n_{3}-n_{2}$ are not pairwise coprime. 
Remark 8. The Kronecker constant does not decay monotonically in $m$ in either cases (ii) or (iii). For example, our computer algorithm shows that

$$
\alpha\{4,5,9\}=5 / 26>1 / 6=\alpha(2,3,7\}=\alpha\{5,6,10\}
$$

and

$$
\alpha\{2,5,7\}=5 / 24>2 / 13=\alpha\{2,5,6\}=\alpha\{2,5,8\} .
$$

Both sets $\{4,5,9\}$ and $\{2,5,7\}$ have the form $\left\{n_{1}, n_{2}, n_{1}+n_{2}\right\}$. By Section 6.3, $\alpha\left\{n_{1}, n_{2}, n_{1}+n_{2}\right\} \geq 1 / 6$ for positive integers $n_{1}$ and $n_{2}$.

\section{Geometric Consequences}

4.1. An upper bound on the Kronecker constant from the geometry. Using our geometric approach we can derive an upper bound on the Kronecker constant of any finite set which is sharp in some (non-trivial) cases.

Theorem 2. Suppose that $P^{(1)}, \ldots, P^{(d-1)}$ are any vectors that span the lattice $\mathcal{K}$. Let $D$ be the diameter of the d-1-dimensional parallelotope generated by the vectors $P^{(j)}$. Then $\alpha\left\{n_{1}, \ldots, n_{d}\right\} \leq D / 2$.

This will be seen to follow almost immediately from elementary geometric reasoning.

Lemma 4. Let $\|\cdot\|$ be any norm on $\mathbb{R}^{n}$ and suppose $\Delta \subseteq \mathbb{R}^{n}$ is a $n$-dimensional parallelotope with diameter $D$. Then for every $g \in \Delta$ there is a vertex $V$ of the parallelotope such that $\|g-V\| \leq D / 2$.

Proof. Without loss of generality 0 is one of the vertices of $\Delta$ and $\Delta$ is spanned by some vectors $P^{(1)}, \ldots, P^{(n)}$. Then $\Delta$ has vertices $\sum_{j=1}^{n} \varepsilon_{j} P^{(j)}$ with $\varepsilon_{j}=0,1$ and $D=\left\|\sum_{j=1}^{n} \delta_{j} P^{(j)}\right\|$ for some choice of $\delta_{j}=0, \pm 1$.

We proceed by induction on $n$. If $n=1$, then $\Delta$ is the line segment $t P^{(1)}$ with $0 \leq t \leq 1$ and it is obvious that the distance from one of $P^{(1)}$ or 0 is at most $\left\|P^{(1)}\right\| / 2=D / 2$.

So assume the result for dimension $n-1$ and put $M=\frac{1}{2} \sum_{j=1}^{n} P^{(j)}$. Note that the line through any vertex, $\sum_{j=1}^{n} \varepsilon_{j} P^{(j)}$, and its diagonally opposite vertex, $\sum_{j=1}^{n}\left(1-\varepsilon_{j}\right) P^{(j)}$, has midpoint $M$, thus $\|M-V\| \leq D / 2$ for any vertex $V$.

Fix any $g \in \Delta, g \neq M$, and let $h$ denote the intersection of the boundary of $\Delta$ with the line segment beginning at $M$ and passing through $g$. Then $h$ belongs to a face of $\Delta$, an $n$-1-dimensional parallelotope whose diameter is at most $D$. By the induction assumption there is a vertex on the face, $V_{0}$, such that $\left\|h-V_{0}\right\| \leq D / 2$, where $\|\cdot\|$ is simply the original norm restricted to the face. But $g$ is a convex combination of $M$ and $h$, say $g=\beta h+(1-\beta) M$, and hence $\left\|g-V_{0}\right\| \leq \beta\left\|h-V_{0}\right\|+$ $(1-\beta)\left\|M-V_{0}\right\| \leq D / 2$.

Proof of Theorem 2. Take $\Delta$ to be the parallelotope spanned by $P^{(1)}, \ldots, P^{(d-1)}$ and \|\|$_{L}$ to be the norm. Then the vertices of $\Delta$ are all in the lattice $\mathcal{K}$ and hence $\alpha_{S}(g) \leq\|g-V\|$ for any $g$ and vertex $V \in \Delta$. To finish the proof, just note that for any $f \in \mathbb{R}^{d-1}, \alpha_{S}(f)=\alpha_{S}(g)$ where $g \in \Delta$ is a suitable translate of $f$.

Example 4. Geometric progressions: Consider the lacunary set $\left\{1, m, \ldots ., m^{k}\right\}$. As we saw in Ex. 1, the lattice $\mathcal{K}$ can be generated by the $k$-tuples,

$$
P^{(j)}=\left(1 / m^{j}, 1 / m^{j-1}, \ldots, 1 / m, 0, \ldots, 0\right), j=1, \ldots, k .
$$


Thus the diameter, $D$, of the $k$-dimensional parallelotope is the maximum of $\|V\|$ where $V=\sum_{j=1}^{k} \delta_{j} P^{(j)}$ and $\delta_{j}=0, \pm 1$. Since only $P^{(s)}, \ldots, P^{(k)}$ have non-zero components in coordinate $s, V=\left(V_{1}, \ldots, V_{k}\right)$ where $V_{s}=\sum_{j=0}^{k-s} \delta_{s+j} / m^{j+1}$. Also,

$$
\|V\|=\max \left\{\left|\frac{m^{k}}{m^{k}+m^{i-1}} V_{i}\right|,\left|\frac{m^{j-1} V_{l}-m^{l-1} V_{j}}{m^{j-1}+m^{l-1}}\right|: i \leq k ; 1 \leq l<j \leq k\right\} .
$$

Straight forward calculations show that

$$
\left|\frac{m^{k}}{m^{k}+m^{i-1}} V_{i}\right| s \leq \frac{1}{m-1}\left(1-\frac{2 m^{i-1}}{m^{k}+m^{i-1}}\right):=B_{i-1, k},
$$

and

$$
\left|\frac{m^{j-1} V_{l}-m^{l-1} V_{j}}{m^{j-1}+m^{l-1}}\right| \leq \frac{1}{m-1}\left(1-\frac{2 m^{l}}{m^{j}+m^{l}}\right):=B_{l, j} .
$$

The maximum of these expressions is $B_{0, k}$. Thus

$$
\alpha\left\{1, m, \ldots, m^{k}\right\} \leq \frac{1}{2(m-1)}\left(1-\frac{2}{m^{k}+1}\right) .
$$

In particular, $\alpha\left\{1, m, m^{2}, \ldots.\right\} \leq 1 / 2(m-1)$, as was shown by other methods in [Graham and Hare 2006a] or [Kunen and Rudin 1999].

Example 5. Two element sets: In the case $d=2$, the lattice $\mathcal{K}$ is spanned by the single vector $P=\operatorname{gcd}\left(n_{1}, n_{2}\right) / n_{2}$. Thus the 1 dimensional parallelotope has diameter $D=\|P\|_{L}=\operatorname{gcd}\left(n_{1}, n_{2}\right) /\left(\left|n_{1}\right|+\left|n_{2}\right|\right)$ and therefore the upper bound of $D / 2$ coincides with the Kronecker constant of $\left\{n_{1}, n_{2}\right\}$.

Example 6. Other sharp examples: The bound of $D / 2$ is also sharp in certain higher dimensional examples, as well. For instance, if $S=\left\{n_{1}, n_{2}, n_{1} n_{2}\right\}$, where $0<n_{1}<n_{2}$ and $\operatorname{gcd}\left(n_{1}, n_{2}\right)=1$, then one can easily check that the lattice $\mathcal{K}$ is spanned by $P=\left(0,1 / n_{1}\right), Q=\left(1 / n_{2}, 0\right)$. The diameter of the associated parallelogram is

$$
\max \{\|P\|,\|Q\|,\|P \pm Q\|\}=\max \left\{\frac{1}{n_{1}+1}, \frac{1}{n_{2}+1}, \frac{2}{n_{1}+n_{2}}\right\} .
$$

If $n_{2} \geq n_{1}+2$, this maximum is $1 /\left(n_{1}+1\right)$, so the proposition implies $\alpha(S) \leq$ $1 / 2\left(n_{1}+1\right)$. This is obviously sharp since it coincides with the Kronecker constant of the two element set $\left\{n_{2}, n_{1} n_{2}\right\}$.

$A$ basis for the lattice associated with the four element set $\left\{ \pm m_{1} m_{2}, m_{1}, m_{2}\right\}$, with $\operatorname{gcd}\left(m_{1}, m_{2}\right)=1$ and $2 \leq m_{1}<m_{2}$, is given by $P_{1}=\left(0,0,1 / m_{1}\right), P_{2}=$ $\left(0,1 / m_{2}, 0\right), P_{3}=(-1,0,0)$. One can verify that the diameter of the three dimensional parallelotope is $1 / 2$ if $m_{1} \geq 3$. Thus $\alpha\left\{-m_{1} m_{2}, m_{1}, m_{2}, m_{1} m_{2}\right\} \leq 1 / 4$ and this is again sharp since the Kronecker constant of the four element set is at least that of the the two element subset, $\left\{-m_{1} m_{2}, m_{1} m_{2}\right\}$, which equals $1 / 4$.

4.2. Points in $\mathbb{R}^{d-1}$ furthest from $\mathbb{Z}^{d-1}$ under an arbitrary norm. Thoughout this section $\|\cdot\|$ will denote an arbitrary norm on $\mathbb{R}^{d-1}$. Given $f \in \mathbb{R}^{d-1}$ we will let $\alpha(f)$ be the distance from $f$ to the integer lattice $\mathbb{Z}^{d-1}$ and denote by $N(f)$ the set of lattice points that are closest to $f$ :

$$
\begin{aligned}
\alpha(f) & =\inf \left\{\|f-v\|: v \in \mathbb{Z}^{d-1}\right\}, \\
N(f) & =\left\{v \in \mathbb{Z}^{d-1}:\|f-v\|=\alpha(f)\right\} .
\end{aligned}
$$


Define

$$
E=\sup \left\{\alpha(f): f \in \mathbb{R}^{d-1}\right\} .
$$

A change of basis argument shows that a special case of this is when $\|\cdot\|=\|\cdot\|_{L}$, in which case $\alpha(f)=\alpha_{S}(f)$ and $E=\alpha(S)$, as defined earlier.

A compactness argument proves that $E=\alpha(f)$ for some $f \in \mathbb{R}^{d-1}$. Furthermore, for all non-empty $F \subseteq \mathbb{Z}^{d-1}$ and $u \in \mathbb{R}^{d-1}$, there is some $w_{0} \in F$ such that

$$
\left\|u-w_{0}\right\|=\inf \{\|u-w\|: w \in F\} .
$$

In particular, for each $f \in \mathbb{R}^{d-1}$, there is some $w_{0} \in \mathbb{Z}^{d-1}$ such that $\alpha(f)=$ $\left\|f-w_{0}\right\|$. Another elementary fact is that $|\alpha(f)-\alpha(g)| \leq\|f-g\|$.

Here we will prove that every norm on $\mathbb{R}^{d-1}$ has the property that there is some $u \in \mathbb{R}^{d-1}$ such that $E=\alpha(u)$ and $N(u)$ has at least $d$ members. This may be known, but we were not able to find a proof in the literature, and it is useful for our computer algorithm. We will also deduce from this that the angular Kronecker constant is always a rational number.

Our argument will be geometric and relies on the notion of supporting hyperplanes: We remind the reader that at each point $p$ of the boundary of a closed, convex set $C$ with non-empty interior there is a supporting hyperplane $H$, i.e., $p \in H=T^{-1}(\lambda)$ where $T: \mathbb{R}^{d-1} \rightarrow \mathbb{R}$ is a linear functional and $T(f) \leq \lambda$ for all $f \in C$. Of course, for all $f, T(f)=f \cdot v$ where $v$ is the normal vector to $H$.

Recall that a norm $\|\cdot\|$ is said to be strictly convex if for all $f, g$ of norm at most one and $t \in(0,1),\|t f+(1-t) g\|<1$; equivalently, the closed unit ball (with respect to this norm) is a strictly convex set. It is easy to see that if $H$ is any supporting hyperplane for the closed unit ball $B$ of a strictly convex norm, then $H \cap B$ is a singleton.

To begin, we will check that most points $u$ cannot satisfy $\alpha(u)=E$.

Lemma 5. Suppose that $N(u)$ has $J$ points, with $J \leq d-1$, say $w_{i}$ for $1 \leq i \leq J$. Let $B_{i}$ be the closed ball centered at $w_{i}$ with radius $\alpha(u)$ and let $H_{i}$ be a supporting hyperplane for $B_{i}$ at $u$, with associated linear transformation $T_{i}$ and normal vector $v_{i}$. If the span of $\left\{v_{i}\right\}_{i=1}^{J}$ has dimension (exactly) $J$, then $\alpha(u)<E$.

Proof. For $v \in F=\mathbb{Z}^{n} \backslash N(u)$, we have $\|u-v\|>\alpha(u)$, thus

$$
\alpha(u)<\inf \{\|u-w\|: w \in F\}=: m .
$$

Set $\delta=\frac{m-\alpha(u)}{2}$ and let $B$ be the open ball of radius $\delta$ centered at $u$. For all $f \in B$ and $w \in F$,

$$
\|f-w\| \geq\|u-w\|-\|f-u\|>m-\delta=\alpha(u)+\delta,
$$

while for $v \in N(u)$

$$
\|f-v\| \leq\|f-u\|+\|u-v\|<\delta+\alpha(u) .
$$

Consequently, for $f \in B, \alpha(f)<\alpha(u)+\delta$ and $N(f) \subset N(u)$.

For each $i$, let $\widetilde{v}_{i}$ be the vector component of $v_{i}$ that is perpendicular to the subspace generated by $\left\{v_{j}\right\}_{j=1}^{J} \backslash\left\{v_{i}\right\}$, meaning that $v_{i}=\widetilde{v}_{i}+x_{i}$ with $x_{i}$ in the linear span of $\left\{v_{j}\right\}_{j=1}^{J} \backslash\left\{v_{i}\right\}$ and $\widetilde{v}_{i} \cdot v_{j}=0$ for $j \neq i$. By the linear independence, $\widetilde{v}_{i} \neq 0$. Moreover,

$$
\widetilde{v}_{i} \cdot v_{i}=\widetilde{v}_{i} \cdot\left(\widetilde{v}_{i}+x_{i}\right)=\widetilde{v}_{i} \cdot \widetilde{v}_{i}>0
$$


For each $i \in\{1, \ldots, J\}$, let $r_{i} \in\left(0, \delta /\left(J\left\|\widetilde{v}_{i}\right\|\right)\right.$ and set $f=u+x$ for $x=\sum_{i=1}^{J} r_{i} \widetilde{v}_{i}$. Then $\|f-u\|=\|x\|<\delta$ and hence $N(f) \subseteq N(u)$ for $f \in B$. Since $\widetilde{v}_{j} \cdot v_{i}=0$ for $j \neq i$, we see that

$$
T_{i}(f)=T_{i}(u)+r_{i} \widetilde{v}_{i} \cdot v_{i}>T_{i}(u) .
$$

As $u \in H_{i}$ and $T_{i}\left(B_{i}\right) \leq T_{i}(u)$, we know that $f \notin B_{i}$ and therefore $\left\|f-w_{i}\right\|>\alpha(u)$ for each $w_{i} \in N(u)$. Thus

$$
E \geq \alpha(f)=\min \left\{\left\|f-w_{i}\right\|: 1 \leq i \leq J\right\}>\alpha(u) .
$$

Next, we prove the desired result for strictly convex norms.

Lemma 6. Any strictly convex norm has the property that there is some $u \in \mathbb{R}^{d-1}$ such that $E=\alpha(u)$ and $N(u)$ has at least $d$ members, $w_{i}, i=1, . ., d$, with the property that for each $i, w_{i} \notin$ convex $\operatorname{hull}\left\{w_{j}: j \neq i\right\}$.

Proof. Choose $u \in \mathbb{R}^{d-1}$ such that $\alpha(u)=E$ and suppose that $N(u)$ has $J \leq d-1$ members, say $w_{i}$ for $1 \leq i \leq J$. Let $B_{i}$ be the closed ball centered at $w_{i}$ with radius $E$ and $H_{i}$ a supporting hyperplane to $B_{i}$ at $u$, with associated linear operator $T_{i}$ and normal vector $v_{i}$. By Lemma 5 , the subspace $V$ spanned by $\left\{v_{i}\right\}_{i=1}^{J}$ has dimension at most $J-1<d-1$ so may choose some non-zero vector $w$, of norm one, such that $w$ is perpendicular to $V$.

If $S=\mathbb{Z}^{d-1} \backslash N(u)$, then $\|u-v\|>E$ for every $v \in S$. Thus $E<\inf \{\|u-v\|$ : $v \in S\}=: m$. Set $z=\frac{m-E}{3} w$ and let $x=u+z$, so that $\|x-u\|=(m-E) / 3$. As in the proof of Lemma 5 , it follows that $N(x) \subset N(u)$. Since $z$ is perpendicular to each $v_{i}$

$$
T_{i}(x)=T_{i}(u)+T_{i}(z)=T_{i}(u)+z \cdot v_{i}=T_{i}(u)
$$

Therefore $x \in H_{i}$. However, $x \neq u$ and $H_{i} \cap B_{i}=\{u\}$, thus $x \notin B_{i}$. Consequently, $\left\|x-w_{i}\right\|>E$. Since this holds for all $i \in\{1, \ldots, J\}$ and $N(x) \subset N(u)$, we have $\alpha(x)>E$ contradicting the definition of $E$.

Thus $N(u)$ contains at least $d$ points $w_{i}$. If there was some $i$ such that $w_{i}=$ $\sum_{j \neq i} \beta_{j} w_{j}$ for $\beta_{j} \geq 0$ and $\sum \beta_{j}=1$, then strict convexity would imply $E=$ $\left\|u-w_{i}\right\|<\sum_{j \neq i} \beta_{j}\left\|u-w_{j}\right\|=E$, which is a contradiction.

We will be able to deduce our result by showing that any norm can be approximated by strictly convex norms.

Lemma 7. For every $\varepsilon>0$ and norm $\|\cdot\|$, there is a strictly convex norm $\|\cdot\|^{\prime}$ such that for all $f \in \mathbb{R}^{d-1}$,

$$
\|f\|^{\prime} \leq\|f\| \leq(1+\varepsilon)\|f\|^{\prime} .
$$

Proof. Let $B$ be the unit ball centered at the origin for $\|\cdot\|$ and consider any point $p$ on the boundary of $B, \partial B$. Let $H=T^{-1}(\lambda)$ be a hyperplane that supports $B$ at $p$, where $T(f)=f \cdot v$ for all $f \in \mathbb{R}^{d-1}$ and $T(f) \leq \lambda$ for all $f \in B$. Without loss of generality we can assume $v$ has Euclidean norm one. As $t v \in B$ for $t>0$ suitably small, it follows that $\lambda>0$.

Let $\delta=\lambda \varepsilon / 2$ and put $\mu=\lambda+\delta$. Let $p^{\prime}=p+\delta v$ and suppose $H^{\prime}=T^{-1}(\mu)$ is the translated hyperplane, $H^{\prime}=H+\delta v$. Note that $T\left(p^{\prime}\right)=\mu$, thus $p^{\prime} \in H^{\prime}$, while $T((1+\varepsilon) p)=(1+\varepsilon) \lambda>\mu$, so $(1+\varepsilon) p$ is on the opposite side of $H^{\prime}$ as is $B$.

For each $p \in \partial B$ consider the (closed) Euclidean ball $S_{p}$ of radius $R_{p}$, centered at $p^{\prime}-R_{p} v$. The point $p^{\prime}$ lies on the boundary of $S_{p}$ and, provided $R_{p}$ is large 
enough, $S_{p} \supseteq B .^{3}$ Because $B=-B$, we also have $B \subseteq-S_{p}$. As $v$ is the normal vector to $H^{\prime}$, it follows that $H^{\prime}$ is a supporting hyperplane for $S_{p}$ at $p^{\prime}$.

Since $T((1+\varepsilon) p)>\mu$, the point $(1+\varepsilon) p \notin S_{p}$. Consequently, the open sets $S_{p}^{c}$ are a cover of the compact set $(1+\varepsilon) \partial B$ and hence there is a finite subcover, say $S_{p_{1}}, \ldots, S_{p_{m}}$. Let

$$
B^{\prime}=\bigcap_{i=1}^{m}\left(S_{p_{i}} \bigcap-S_{p_{i}}\right)
$$

The construction ensures that $(1+\varepsilon) \partial B \cap B^{\prime}$ is empty and clearly $B \subseteq B^{\prime}$. As $0 \in B^{\prime}$, a convexity argument implies $B^{\prime} \subseteq(1+\varepsilon) B$.

Since $B^{\prime}$ is closed, symmetric, convex and has 0 in its interior, there is a norm $\|\cdot\|^{\prime}$ for which $B^{\prime}$ is the unit ball. Because $B \subseteq B^{\prime} \subseteq(1+\varepsilon) B$, for all $f \in \mathbb{R}^{d-1}$ we have $\|f\|^{\prime} \leq\|f\| \leq(1+\varepsilon)\|f\|^{\prime}$. The strict convexity of this norm follows from the strict convexity of the Euclidean balls, $S_{p}$ and $-S_{p}$.

Theorem 3. Every norm has the property that there is some $u \in \mathbb{R}^{d-1}$ such that $E=\alpha(u)$ and $N(u)$ has at least $d$ members, $w_{i}, i=1, . ., d$, with the property that for each $i, w_{i} \notin$ convex hull $\left\{w_{j}: j \neq i\right\}$.

Proof. Using the previous lemma we can obtain strictly convex norms, $\|\cdot\|_{n}$, such that $\|f\|_{n} \leq\|f\| \leq(1+1 / n)\|f\|_{n}$. For the norm $\|\cdot\|_{n}$, let $E_{n}$ correspond to $E, \alpha_{n}$ correspond to $\alpha$ and $N_{n}$ correspond to $N$.

Given any $f \in \mathbb{R}^{d-1}$ let $w_{0} \in \mathbb{Z}^{d-1}$ be such that $\alpha_{n}(f)=\left\|f-w_{0}\right\|$. Then

$$
\alpha(f) \leq\left\|f-w_{0}\right\| \leq(1+1 / n)\left\|f-w_{0}\right\|_{n}=(1+1 / n) \alpha_{n}(f) .
$$

Similarly,

$$
\alpha(f) \geq \alpha_{n}(f) .
$$

As these inequalities hold for all $f$, it follows that

$$
(1+1 / n)^{-1} E \leq E_{n} \leq E
$$

and hence $E_{n} \rightarrow E$. In particular, $\left\{E_{n}\right\}_{n}$ is bounded.

Since $\|\cdot\|_{n}$ is strictly convex, Lemma 6 implies that there is some $u_{n} \in \mathbb{R}^{d-1}$ such that $\alpha_{n}\left(u_{n}\right)=E_{n}$ and $N_{n}\left(u_{n}\right)$ has at least $d$ elements of $\mathbb{Z}^{d-1}$. The $\mathbb{Z}^{d-1}$ periodicity of $\alpha_{n}$ ensures that we can assume $u_{n} \in[0,1]^{d-1}$. By passing to a subsequence, if necessary, we can assume $u_{n} \rightarrow u \in[0,1]^{d-1}$ (in $\|\cdot\|$ norm). Now

$$
\begin{aligned}
\left|\alpha(u)-\alpha_{n}\left(u_{n}\right)\right| & \leq\left|\alpha(u)-\alpha\left(u_{n}\right)\right|+\left|\alpha\left(u_{n}\right)-\alpha_{n}\left(u_{n}\right)\right| \\
& \leq\left\|u-u_{n}\right\|+(1 / n) \alpha_{n}\left(u_{n}\right) \\
& \leq\left\|u-u_{n}\right\|+(1 / n) E_{n} \rightarrow 0
\end{aligned}
$$

and thus $\alpha(u)=E$.

For each $n$, let $v_{n, j}, 1 \leq j \leq d$, be $d$ distinct members of $\mathbb{Z}^{d-1}$, having the convexity property and satisfying

$$
E_{n}=\left\|u_{n}-v_{n, j}\right\|_{n} \text { for } 1 \leq j \leq d .
$$

\footnotetext{
${ }^{3}$ In fact, if $\pi(B)$ denotes the Euclidean projection of $B$ onto $H^{\prime}, \tau$ is the maximum Euclidean distance of any point in $\pi(B)$ from $p^{\prime}$ and $d \geq \max (\tau, \delta)$ satisfies $\left(d^{2}+\delta^{2}\right) / 2 \delta \geq-\delta+2 \mu$, then $R_{p}=\left(d^{2}+\delta^{2}\right) / 2 \delta$ will suffice.
} 
Since all norms are equivalent on $\mathbb{R}^{d-1}$ there exists $C$ such that $\|z\| \leq C$ for all $z \in[0,1]^{d-1}$. Thus

$$
\begin{aligned}
\left\|v_{n, j}\right\| & \leq\left\|u_{n}-v_{n, j}\right\|+\left\|u_{n}\right\| \\
& \leq(1+1 / n)\left\|u_{n}-v_{n, j}\right\|_{n}+C \\
& \leq(1+1 / n) E_{n}+C<\infty .
\end{aligned}
$$

Because $\mathbb{Z}^{d-1}$ has only finitely many members in any bounded set, there is a subsequence $v_{n_{k}, 1}$ which is constant, say $v_{1}$. But then a subsequence of $v_{n_{k}, 2}$ is constant, say $v_{2}$. By repeated application of this argument we can assume, without loss of generality, that for each $j=1, \ldots, d$, the sequence $v_{n, j}$ is identically $v_{j}$. Thus $v_{1}, \ldots, v_{d}$ are distinct and even satisfy the convexity condition. Moreover, the relationship between the two norms and the fact that $v_{n, j} \in N\left(u_{n}\right)$ implies that

$$
E_{n} \leq\left\|u_{n}-v_{n, j}\right\| \leq(1+1 / n) E_{n}
$$

and therefore

$$
\left\|u-v_{j}\right\|=\lim _{n \rightarrow \infty}\left\|u_{n}-v_{n, j}\right\|=E .
$$

Thus these $d$ points, $v_{j}$, all belong to $N(u)$.

One application of this result is to show that the angular Kronecker constant is always a rational number.

Corollary 4. For any finite set $S \subset \mathbb{Z}$, the angular Kronecker constant of $S$ is a rational number.

Proof. If $0 \in S$, then $\alpha(S)=1 / 2$, so assume that $0 \notin S$ and that $S$ has $d$ elements. Applying the previous theorem, it follows that there is some $f \in \mathbb{R}^{d-1} \times\{0\}$ and at least $d$ distinct $w_{i}$ belonging to the associated lattice $\mathcal{K}$ such that $\alpha(S)=\alpha_{S}(f)=$ $\left\|f-w_{i}\right\|_{L}$ for $i=1, \ldots, d$. Since any ball of fixed radius $\alpha(S)$ contains a bounded number of elements of $\mathcal{K}$, there is a maximum integer $n \geq d$ for which there is some $f \in \mathbb{R}^{d-1} \times\{0\}$ and $n$ distinct members $w_{i} \in \mathcal{K}$ such that

$$
\alpha(S)=\alpha_{S}(f)=\left\|f-w_{i}\right\|_{L} \quad \text { for } 1 \leq i \leq n .
$$

By Proposition 1 the norm \|\|$_{L}$ is given by a set of vectors $\mathcal{V}=\left\{v_{1}, \ldots, v_{d(d-1)}\right\}$ such that

$$
\|u\|_{L}=\max \left\{v_{i} \cdot u: i=1, \ldots, d(d-1)\right\}
$$

Suppose that $v_{i} \in \mathcal{V}$ satisfies $\left\|f-w_{i}\right\|_{L}=v_{i} \cdot\left(f-w_{i}\right)$. Having this equal to $\alpha(S)$ is equivalent to requiring:

$\left(*_{i}\right) \quad v_{i} \cdot\left(f-w_{i}\right) \geq v \cdot\left(f-w_{i}\right)$ for all $v \in \mathcal{V}$ and $v_{i} \cdot\left(f-w_{i}\right)=\alpha(S)$.

Let $C$ consist of all $g \in \mathbb{R}^{d-1} \times\{0\}$ such that $\left(*_{i}\right)$ holds for all $1 \leq i \leq n$, with $g$ in the role of $f$. Clearly $f \in C$ and $C$ is compact and convex. The extreme points of $C$ are determined by the linear constraints that define $C$, and as each $w_{i}$ and $v \in \mathcal{V}$ has rational components, any extreme point has rational components.

Let $h$ be an extreme point of $C$. If $\alpha_{S}(h)=\alpha(S)$, then, of course, $v_{i} \cdot\left(h-w_{i}\right)=$ $\alpha(S)$ implies that $\alpha(S)$ is rational.

So suppose $\alpha_{S}(h)<\alpha(S)$ and put $g_{t}=(1-t) f+t h$ for $0 \leq t \leq 1$. Since $C$ is convex, $g_{t} \in C$ and thus $\left\|g_{t}-w_{i}\right\|=\alpha(S)$ for all $1 \leq i \leq n$. Put $t_{0}=\sup \{t \in[0,1]$ $\left.: \alpha_{S}\left(g_{t}\right)=\alpha(S)\right\}$. By continuity and compactness, $\alpha_{S}\left(g_{t_{0}}\right)=\alpha(S)$, thus $t_{0}<1$. For each $t \in\left(t_{0}, 1\right]$ there is some $u_{t} \in \mathcal{K} \backslash\left\{w_{1}, \ldots, w_{n}\right\}$ such that

$$
\left\|g_{t}-u_{t}\right\|_{L}=\alpha_{S}\left(g_{t}\right)<\alpha(S) .
$$


Within an $\alpha(S)$ neighborhood of $[f, h]$, there are at most finitely many members of $\mathcal{K}$. Hence there is some $u \in \mathcal{K} \backslash\left\{w_{1}, \ldots, w_{n}\right\}$ and sequence $t_{i}$ decreasing to $t_{0}$ such that $u_{t_{i}}=u$ for all $i$. By continuity

$$
\left\|g_{t_{0}}-u\right\|_{L}=\lim _{i \rightarrow \infty}\left\|g_{t_{i}}-u\right\|_{L} \leq \alpha(S) .
$$

By the definition of $\alpha, \alpha(S)=\alpha_{S}\left(g_{t_{0}}\right) \leq\left\|g_{t_{0}}-u\right\|$, so we must have $\left\|g_{t_{0}}-u\right\|_{L}=$ $\alpha(S)$. This violates the maximality condition that defines $n$.

\section{The Geometry Problem in $\mathbb{R}^{3}$}

Here we make the $\ell^{\infty}$ geometry and the calculation of the angular Kronecker constant more explicit in the case of $\mathbb{R}^{3}$.

Theorem 4. Let $S=\left\{n_{1}, n_{2}, n_{3}\right\} \subset \mathbb{Z}^{3} \backslash\{0\}$ with $\operatorname{gcd}\left\{n_{1}, n_{2}, n_{3}\right\}=1$ and let $m_{2}=\operatorname{gcd}\left(n_{2}, n_{3}\right)$. Let $\mathcal{K}$ be the group associated with the vector $n=\left(n_{1}, n_{2}, n_{3}\right)$, as described in section 2.

(i) We can choose generators, $P, Q$ of $\mathcal{K}$, such that $P=\left(r /\left|n_{3}\right|, m_{2} /\left|n_{3}\right|, 0\right)$ and $Q=\left(1 / m_{2}, 0,0\right)$ where $r \equiv t n_{1} \bmod n_{3}$ and $t$ is any integer satisfying $t n_{2} \equiv$ $m_{2} \bmod n_{3}$. Moreover, $r$ can be chosen with $0 \leq r /\left|n_{3}\right|<1 / m_{2}$.

(ii) Suppose that $P=\left(r /\left|n_{3}\right|, m_{2} /\left|n_{3}\right|, 0\right)$ and $Q=\left(1 / m_{2}, 0,0\right)$ generate $\mathcal{K}$ as a group. The number $\alpha(S)$ is the smallest number $E$ such that, for all $x, y \in \mathbb{R}$, there are integers $s, t$ for which

$$
\begin{aligned}
(2,3): & \frac{\left|n_{3}\right|}{\left|n_{2}\right|+\left|n_{3}\right|}\left|\frac{t m_{2}}{n_{3}}-y\right| \leq E \\
(1,3): & \frac{\left|n_{3}\right|}{\left|n_{1}\right|+\left|n_{3}\right|}\left|\frac{t r}{n_{3}}+\frac{s}{m_{2}}-x\right| \leq E \\
(1,2) & : \quad \frac{\left|n_{2}\left(\frac{t r}{n_{3}}+\frac{s}{m_{2}}-x\right)-n_{1}\left(\frac{t m_{2}}{n_{3}}-y\right)\right|}{\left|n_{1}\right|+\left|n_{2}\right|} \leq E .
\end{aligned}
$$

Proof. (i) By Prop. 4 there are generators $Q:=P^{(1)}$ and $P:=P^{(2)}$ for $\mathcal{K}$ such that $Q=\left(1 / m_{2}, 0,0\right)$ and $P=\left(P_{1}, m_{2} /\left|n_{3}\right|, 0\right)$ with $P_{1} \in[0,1)$. As $P \in \mathcal{K}$, Lemma 3 implies $P \bmod 1=\left(r /\left|n_{3}\right|\right) n \bmod 1$ for some $r \in \mathbb{Z}$. Thus $P_{2}=m_{2} /\left|n_{3}\right| \equiv r n_{2} /\left|n_{3}\right|$ and $P_{1} \equiv r n_{1} /\left|n_{3}\right|$, as claimed.

Now let $\beta$ be an integer such that $\beta / m_{2} \leq r /\left|n_{3}\right|<(\beta+1) / m_{2}$ and let $\tilde{P}=$ $P-\beta Q$. Then $(\tilde{P})_{1} \in\left[0,1 / m_{2}\right), \tilde{P}_{2}=m_{2} / n_{3}$, and $\tilde{P}_{3}=0$. Because $m_{2}$ divides $n_{3}$, there is some integer $r^{\prime}$ such that $\tilde{P}_{1}=r /\left|n_{3}\right|-\beta / m_{2}=r^{\prime} /\left|n_{3}\right|$. By Prop. $3, Q$ and $\tilde{P}$ will also generate $\mathcal{K}$.

(ii) Since $\alpha(S)=\alpha(-S)$, we can assume $n_{3}>0$. Any $k \in \mathcal{K}$ can be written as $k=k(s, t)=s Q+t P$ for some integers $s$ and $t$, where $P, Q$ are chosen as above. By Theorem 1,

$$
\alpha(S)=\sup _{(x, y, 0) \in \mathbb{R}^{3}}\left(\inf _{s, t \in \mathbb{Z}}\left[\max _{1 \leq i<j \leq 3}\left\{\frac{\left|n_{j}\left(x_{i}-k(s, t)_{i}\right)-n_{i}\left(x_{j}-k(s, t)_{j}\right)\right|}{\left|n_{i}\right|+\left|n_{j}\right|}\right\}\right]\right) .
$$

Because

$$
k(s, t)_{1}=s / m_{2}+t r / n_{3}, \quad k(s, t)_{2}=t m_{2} / n_{3} \text { and } k(s, t)_{3}=0,
$$

we have

$$
\frac{\left|n_{2}\left(x-s / m_{2}-t r / n_{3}\right)-n_{1}\left(y-t m_{2} / n_{3}\right)\right|}{\left|n_{1}\right|+\left|n_{2}\right|}=\frac{\left|n_{2}\left(\frac{t r}{n_{3}}+\frac{s}{m_{2}}-x\right)+n_{1}\left(\frac{t m_{2}}{n_{3}}-y\right)\right|}{\left|n_{1}\right|+\left|n_{2}\right|},
$$




$$
\frac{\left|n_{3}\left(x-s / m_{2}-t r / n_{3}\right)\right|}{\left|n_{1}\right|+\left|n_{3}\right|}=\frac{\left|n_{3}\right|}{\left|n_{1}\right|+\left|n_{3}\right|}\left|\frac{\operatorname{tr}}{n_{3}}+\frac{s}{m_{2}}-x\right|,
$$

and

$$
\frac{\left|n_{3}\left(y-t m_{2} / n_{3}\right)\right|}{\left|n_{2}\right|+\left|n_{3}\right|}=\frac{\left|n_{3}\right|}{\left|n_{2}\right|+\left|n_{3}\right|}\left|\frac{t m_{2}}{n_{3}}-y\right| .
$$

Since the infimum over $s, t \in \mathbb{Z}$ is clearly attained, this completes the proof.

Example 7. Let $S=\{-a, a, b\}$ with $-a<a<b$ and $\operatorname{gcd}(a, b)=1$. Applying Prop. 4, we see there are generators $P:=P^{(1)}$ and $Q:=P^{(2)}$ for $\mathcal{K}$ such that $Q=(1,0,0)$ and $P_{2}=1 / b$. If $t a=1 \bmod b$, then $t(-a)=-1 \bmod b=(b-1) \bmod b$. Thus we could take $P=(-1 / b, 1 / b, 0)$ or $((b-1) / b, 1 / b, 0)$.

Example 8. Let $S=\{a, a+b, a+2 b\}$ for positive integers $a$ and $b$ with $\operatorname{gcd}(a, b)=1$. Then $\operatorname{gcd}(a, a+b)=\operatorname{gcd}(a+b, a+2 b)=1$. We have generators $P:=P^{(1)}$ and $Q:=P^{(2)}=(1,0,0)$ for $\mathcal{K}$ with $P_{2}=1 /(a+2 b)$. If $t(a+b)=1 \bmod (a+2 b)$, then $2 t(a+b)=2 \bmod (a+2 b)$. As $2 t(a+b)=t a+t(a+2 b)$, it follows that $t a=2 t(a+b)=2 \bmod (a+2 b)$, and therefore we can let $P=(2,1,0) /(a+2 b)$.

We can also explicitly determine which of the equations $\left|n_{j} u_{i}-n_{i} u_{j}\right| /\left(\left|n_{i}\right|+\left|n_{j}\right|\right)$ is maximal in the case $d=3$ and this is useful for understanding the unit ball of the quotient norm $\|\cdot\|_{L}$.

We will assume the integers $n_{1}, n_{2}, n_{3}$ satisfy $0<n_{1}<n_{2}<n_{3}$. As usual, let $L=\{x n: x \in \mathbb{R}\}$.

Lemma 8. Let $s_{1}=\left(n_{2}+n_{3}\right) /\left(n_{1}+n_{3}\right), s_{2}=\left(n_{2}-n_{3}\right) /\left(n_{1}+n_{3}\right)$ and $s_{3}=$ $\left(n_{2}+n_{3}\right) /\left(n_{1}-n_{3}\right)$. For $(u, v) \in \mathbb{R}^{2}$,

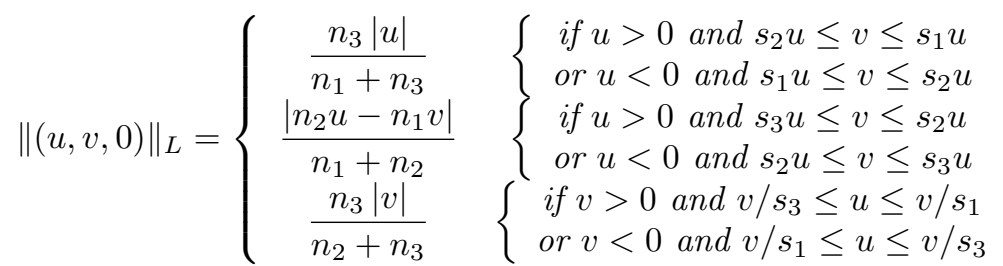

The proof is elementary and is left for the reader. Note that $s_{1}>1, s_{2} \in(-1,0)$ and $s_{3}<-1$.

The mapping $\phi((u, v))=(u, v, 0)-L$ is a linear isomorphism from $\mathbb{R}^{2}$ onto $\mathbb{R}^{3} / L$ and we can lift the quotient norm from $\mathbb{R}^{3} / L$ to $\mathbb{R}^{2}$ by setting $\|(u, v)\|=\|(u, v, 0)\|_{L}$. Let $r_{1}=\left(n_{1}+n_{3}\right) / n_{3}, r_{2}=\left(n_{2}+n_{3}\right) / n_{3}, r_{3}=\left(n_{1}+n_{2}\right) / \sqrt{n_{1}^{2}+n_{2}^{2}}$ and let $s_{i}$ be defined as in Lemma 8 . We can now describe the shape of a unit ball for the quotient space $\mathbb{R}^{3} / L$.

Proposition 7. (i) The unit ball for this metric on $\mathbb{R}^{2}$ is a hexagon, the convex hull of these six points which are enumerated clockwise:

$$
\begin{aligned}
& \left.P_{1}=\left(r_{1}, r_{2}\right)=-P_{4} \text { (on the line through the origin with slope } s_{1}\right) \text {, } \\
& \left.P_{2}=\left(r_{1},\left(n_{2}-n_{3}\right) / n_{3}\right)=-P_{5} \text { (on the line through the origin with slope } s_{2}\right) \text {, } \\
& \left.P_{3}=\left(\left(n_{3}-n_{1}\right) / n_{3},-r_{2}\right)=-P_{6} \text { (on the line through the origin with slope } s_{3}\right) \text {. }
\end{aligned}
$$

(ii) The area of the unit ball is $4\left(n_{1}+n_{2}+n_{3}\right) / n_{3}$.

Proof. The reader can verify that the hexagon shape of the unit ball, with the specified vertices, follows from the previous lemma. 
A formula for the area $A$ of a polygon in terms of the coordinates of its vertices $P_{i}=\left(x_{i}, y_{i}\right)_{i=1}^{n}$ is

$$
A=\left|\frac{1}{2} \sum_{i=1}^{n}\left(x_{i} y_{i+1}-x_{i+1} y_{i}\right)\right| \quad \text { with }\left(x_{n+1}, y_{n+1}\right) \text { set equal to }\left(x_{1}, y_{1}\right)
$$

[Beyer 1987]. Applying this formula gives the stated value for the area of the unit ball.

From these geometric ideas, we can also obtain a lower bound in the case of finite sets of size three.

Proposition 8. If $1 \leq n_{1}<n_{2}<n_{3}, \operatorname{gcd}\left(n_{1}, n_{2}, n_{3}\right)=1$ and $S=\left\{n_{1}, n_{2}, n_{3}\right\}$, then

$$
\alpha(S) \geq \frac{1}{\sqrt{8\left(n_{1}+n_{2}+n_{3}\right)}} .
$$

Proof. Assume $\alpha(S)=\alpha_{S}(f)$. By Theorem 3 there are at least three lattice points $w_{i}, i=1,2,3$, which are not co-linear and have the property that $\alpha_{S}(f)=\left\|f-w_{i}\right\|$. If $w$ is any other lattice point in the convex hull of $w_{1}, w_{2}, w_{3}$, then the fact that $\alpha_{S}(f)=\left\|f-w_{i}\right\|$ and the triangle inequality ensures that $w$ also satisfies $\alpha_{S}(f)=\|f-w\|$. Consequently, there is no loss of generality in assuming that the lattice points $w_{i}$ are not co-linear and no other lattice point lies in their convex hull. This latter condition implies that the three points generate the lattice.

All parallelograms spanned by three generating points of the lattice have the same area and therefore these points span a parallelogram of area $1 / n_{3}$ (Cor. 1 ).

The convex hull of the three points is a triangle, $T$, whose area is half that of the parallelogram. This triangle is contained inside the closed ball, $B$, centered at $f$ with radius $\alpha(S)$. Using the previous theorem it follows that

$$
\begin{aligned}
\frac{1}{2 n_{3}} & =\operatorname{Area}(T) \leq \operatorname{Area}(B) \\
& =\alpha(S)^{2}\left(\frac{4\left(n_{1}+n_{2}+n_{3}\right)}{n_{3}}\right)
\end{aligned}
$$

from which we derive the lower bound stated in the proposition.

\section{Kronecker constants For three ELEMENT SETS}

In this section we will use Theorem 4 to calculate (or bound) $\alpha(S)$ for various three element sets.

6.1. Mixed positive and negative integers. We begin with the case when the integers $\left|n_{1}\right|,\left|n_{2}\right|,\left|n_{3}\right|$ are not distinct and for this case we are able to give a complete solution.

When $\left|n_{1}\right|,\left|n_{2}\right|,\left|n_{3}\right|$ are distinct the problem of interpolating angles, $t_{1}, t_{2}, t_{3}$, at $n_{1}, n_{2}, n_{3}$ is effectively the same as the problem of interpolating $\pm t_{1}, \pm t_{2}, \pm t_{3}$ at $\pm n_{1}, \pm n_{2}, \pm n_{3}$ and therefore $\alpha\left\{n_{1}, n_{2}, n_{3}\right\}=\alpha\left\{\left|n_{1}\right|,\left|n_{2}\right|,\left|n_{3}\right|\right\}$. Thus later in this section we will typically assume either that all $n_{j}>0$ or choose the signs as is convenient.

Proposition 9. Suppose $a, b>0$ are coprime integers.

(i) Assume $b<a$. Then $\alpha\{-a, b, a\}=1 / 4$. 
(ii) Assume $b>a$. If $a \geq 2$, then $\alpha\{-a, a, b\}=1 / 4$ and

$$
\alpha\{-1,1, b\}= \begin{cases}\frac{b+2}{4(b+1)} & \text { if } b \text { is even } \\ \frac{b+1}{2(2 b+1)} & \text { if } b \text { is odd }\end{cases}
$$

Proof. (i) Let $n=(-a, b, a)$, the order in which we list $\{-a, b, a\}$. For this ordering, we have $P=(0,1 / a)$ and $Q=(1,0)$ because $k(-a) \equiv 0 \bmod a$ for all integers $k$. Thus $\alpha\{-a, b, a\}$ will be the minimum value $E$ such that for each $x, y$ there are integers $r, s$ such that all of $(1,3),(2,3)$ and $(1,3)$ are bounded above by $E$, with

$$
\begin{aligned}
(1,3) & :=\frac{|a(x-s)|}{|-a|+|a|}=\frac{|s-x|}{2} \\
(2,3) & :=\frac{|a(y-r / a)|}{|a|+|b|}=\frac{|r-a y|}{a+b} \text { and } \\
(1,2) & :=\frac{|b(x-s)-(-a)(y-r / a)|}{|-a|+|b|}=\frac{|r-a y+b(s-x)|}{a+b}
\end{aligned}
$$

Clearly, if $x=1 / 2$, the inequality $(1,3) \leq E$ can only be satisfied with $E \geq 1 / 4$, so it is enough to show that $E=1 / 4$ suffices.

Given any $x$ there is a choice of integer $s$ such that $|s-x| \leq 1 / 2$, which implies $(1,3) \leq 1 / 4$.

Inequality $(2,3) \leq 1 / 4$ holds if and only if $|r-a y| \leq(a+b) / 4$. If $a+b \geq 4$, then for any fixed $y$ there are at least two choices of integers for $r$ which could be used, the nearest integers $r$ to ay satisfying $r-a y>0$ or $r-a y<0$. Take the choice of $r$ with $\operatorname{sign}(r-a y)=-\operatorname{sign}(s-x)$. Then $(1,2) \leq 1 / 4$, in part because $b<a$ :

$$
\frac{|r-a y+b(s-x)|}{a+b} \leq \max \left(\frac{|r-a y|}{a+b}, \frac{b|s-x|}{a+b}\right) \leq \max \left(\frac{1}{4}, \frac{b / 2}{b+b}\right)=\frac{1}{4}
$$

Otherwise, $b=1$ and $a=2$. If $a y \in[1 / 4,3 / 4] \bmod 1$, we make the same two choices for $r$ but with $|r-a y| \leq 3 / 4$. The same argument works because $|r-a y| /(a+b) \leq(3 / 4) / 3=1 / 4$. Otherwise, choose $r$ such that $|r-a y| \leq 1 / 4$. Then

$$
(1,2) \leq \frac{|r-a y|}{a+b}+\frac{b|s-x|}{a+b} \leq \frac{1 / 4+b / 2}{a+b}=\frac{1}{4} .
$$

(ii) Let $n=(-a, a, b)$ be the order in which we list $S=\{-a, a, b\}$. The distinction between $b<a$ and $b>a$ arises because of the definition of $P$. We may take $P_{2}=1 / b$ and to determine $P_{1}$ we observe that if $k a \equiv 1 \bmod \mathrm{b}$, then $-k a \equiv-1 \bmod \mathrm{b}$, so we may take $P_{1}=-1 / b$. As before, $Q=(1,0) . \alpha(S)$ is the smallest constant $E$ such that

$$
\begin{aligned}
& \text { for }(1,3): \quad \frac{b|-r / b+s-x|}{a+b} \leq E \\
& \text { for }(2,3) \quad: \quad \frac{b|r / b-y|}{a+b} \leq E, \text { and } \\
& \text { for }(1,2) \quad: \quad \frac{|-a(r / b-y)-a(-r / b+s-x)|}{2 a} \\
& \quad=\frac{|-y-x+s|}{2} \leq E .
\end{aligned}
$$

If $(-x-y) \bmod 1=1 / 2$, then $(1,2)=1 / 4$ and we must have $E \geq 1 / 4$. 
If we put $\Delta_{r}=y-r / b$ and choose $\beta \in[0,1]$ congruent to $(-x-y) \bmod 1$, we may rewrite these as (with a possibly different integer $s$ )

$$
\begin{aligned}
&(1,3)^{\prime}: \quad \frac{b\left|\Delta_{r}+\beta+s\right|}{a+b} \leq E \\
&(2,3)^{\prime}: \quad \frac{b\left|\Delta_{r}\right|}{a+b} \leq E, \text { and } \\
&(1,2)^{\prime} \quad: \quad \frac{|\beta+s|}{2} \leq E .
\end{aligned}
$$

First we will show that if we take $E$ equal to the claimed value for $\alpha(S)$, then all three inequalities are satisfied.

Recall that $\alpha_{S}((-x,-y, 0))=\alpha_{S}((x, y, 0))$. If $\beta \neq 0$, we have $1-\beta=(x+$ $y) \bmod 1$. If $\beta=0$, of course $(x+y) \bmod 1=\beta$. So, without losing generality, we may assume $\beta \leq 1 / 2$. Since $E \geq 1 / 4$, that puts $\beta \in[0,2 E]$. Because $E<1 / 2$, we must use $s=0$ or $s=-1$ to satisfy $(1,2)^{\prime}$.

Suppose that $\beta \leq 1-2 E$. We take $s=0$ to satisfy $(1,2)^{\prime}$. Then we need to choose $r$ such that

$$
-E\left(1+\frac{a}{b}\right) \leq \Delta_{r} \leq E\left(1+\frac{a}{b}\right)-\beta
$$

to satisfy both $(1,3)^{\prime}$ and $(2,3)^{\prime}$. With $\beta \leq 1-2 E$, the interval above has length

$$
2 E\left(1+\frac{a}{b}\right)-\beta \geq E\left(4+\frac{2 a}{b}\right)-1 \geq \frac{1}{b}
$$

(for any of the three possibilities for $E$ ). This is long enough to ensure that, for each $y$, there is some integer $r$ such that $\Delta_{r}=y-r / b$ is in this interval.

If $\beta \in[1-2 E, 1 / 2]$, we can let $s=-1$ or $s=0$ to satisfy $(1,2)^{\prime}$. If we use $s=-1$, then we need to choose $r$ such that

$$
-E\left(1+\frac{a}{b}\right)+1-\beta \leq \Delta_{r} \leq E\left(1+\frac{a}{b}\right)
$$

to satisfy $(1,3)^{\prime}$ and $(2,3)^{\prime}$. Depending on whether $s=0$ or $s=-1$, we will need to choose an integer $r$ so that $\Delta_{r}$ is in either

$$
\left[-E\left(1+\frac{a}{b}\right), E\left(1+\frac{a}{b}\right)-\beta\right]=\left[I_{1}, I_{2}\right]
$$

or

$$
\left[-E\left(1+\frac{a}{b}\right)+1-\beta, E\left(1+\frac{a}{b}\right)\right]=\left[J_{1}, J_{2}\right]
$$

The sum of the lengths of these intervals is $4 E(1+a / b)-1$. If $E=1 / 4$ and $a \geq 2$, this sum of lengths is at least $2 / b$. So one of the two intervals will have length at least $1 / b$ and hence will contain $\Delta_{r}$ for some integer $r$. This is also true if $a=1$ and $E=\frac{b+2}{4(b+1)}$ (the $b$ even case).

Otherwise, suppose $b$ is odd, say $b=2 m+1$ (and $a=1$ ). With the specified value for $E$, the sum of the lengths of intervals $\left[I_{1}, I_{2}\right]$ and $\left[J_{1}, J_{2}\right]$ is $(3 b+2) /\left(2 b^{2}+b\right)$, which is greater than $1 / b$, but not as much as $2 / b$. Note that

$$
\begin{aligned}
J_{2}-I_{1} & =2 E\left(1+\frac{1}{b}\right)=\frac{(1+b)^{2}}{b(2 b+1)} \\
& =\frac{m+1}{b}+\frac{m+1}{b(2 b+1)} .
\end{aligned}
$$


Put $I_{k}^{\prime}=I_{k}+(1+m) / b$, so that

$$
J_{2}-I_{1}^{\prime}=\frac{(m+1)}{b(2 b+1)} .
$$

If $J_{1}>I_{1}^{\prime}$, then

$$
\text { length }\left[J_{1}, J_{2}\right] \leq \frac{(m+1)}{b(2 b+1)} \text {, }
$$

from which it follows that the length of $\left[I_{1}, I_{2}\right]$ is at least

$$
\frac{3 b+2}{b(2 b+1)}-\frac{m+1}{b(2 b+1)}=\frac{5 m+4}{b(4 m+3)} \geq \frac{1}{b}
$$

and therefore we can choose an integer $r$ so that $\Delta_{r}=y-r / b \in\left[I_{1}, I_{2}\right]$.

If not, then $J_{1} \leq I_{1}^{\prime} \leq J_{2}$. Note that

$$
\beta \leq 1 / 2 \quad \Rightarrow \quad-\beta+\frac{m+1}{b} \geq \frac{\frac{1}{2}}{b}>0,
$$

and thus $I_{2}^{\prime}>J_{2}$. The length of the interval $\left[J_{1}, I_{2}^{\prime}\right]$ is the sum of the lengths of the intervals $\left[I_{1}, I_{2}\right]$ and $\left[J_{1}^{\prime}, J_{2}^{\prime}\right]$, less the length of their overlap. Since the overlap has length $J_{2}-I_{1}^{\prime}=(m+1) / b(2 b+1)$, the same calculation as in (3) shows that the length of $\left[J_{1}, I_{2}^{\prime}\right]$ is also at least $1 / b$. For any $y$, we can find an integer $t$ with $t / b-y$ in one of the intervals $\left[I_{1}^{\prime}, I_{2}^{\prime}\right]$ or $\left[J_{1}, J_{2}\right]$. In the former case, taking $r=t-1-m$, we get $\Delta_{r} \in\left[I_{1}, I_{2}\right]$.

This completes the proof that the specified $E$ is an upper bound for the angular Kronecker constant.

As we have already mentioned, $(1,2) \leq E$ requires $E \geq 1 / 4$. So, to show the sharpness of $E$ we only need to consider the case $a=1$.

First, suppose $b$ is odd, put $(x, y)=(E, E)$ and assume the angular Kronecker constant is $E_{\varepsilon}=E-\varepsilon$ for some $\varepsilon>0$. Here $1 / 4<E<1 / 2$ and therefore $-1<-2 E<-1 / 2$. The inequality $(1,2) \leq E_{\epsilon}$ simplifies to $|-2 E+s| / 2 \leq E_{\varepsilon}$ and this can only be satisfied with $s=1$. With $s=1$, inequalities $(1,3) \leq E_{\epsilon}$ and $(2,3) \leq E_{\epsilon}$ become

$$
\frac{b}{1+b}\left|\frac{-r}{b}+1-E\right| \leq E-\varepsilon \frac{b}{1+b}\left|\frac{r}{b}-E\right| \leq E-\varepsilon
$$

and together these require

$$
1-E\left(\frac{1+2 b}{b}\right)+\varepsilon\left(\frac{b+1}{b}\right) \leq \frac{r}{b} \leq E\left(\frac{1+2 b}{b}\right)-\varepsilon\left(\frac{b+1}{b}\right) .
$$

As $E(1+2 b)=(1+b) / 2$, this simplifies to

$$
\frac{b-1}{2 b}+\varepsilon\left(\frac{b+1}{b}\right) \leq \frac{r}{b} \leq \frac{1+b}{2 b}-\varepsilon\left(\frac{b+1}{b}\right) .
$$

Since $b$ is odd, $(b-1) / 2$ and $(b+1) / 2$ are consecutive integers, so this inequality cannot hold for any integer $r$ and $\varepsilon>0$.

For $b$ even we take

$$
(x, y)= \begin{cases}\left(\frac{1}{2}, 0\right) & \text { if } b \equiv 2 \bmod 4 \\ \left(\frac{b-1}{2 b}, \frac{1}{2 b}\right) & \text { if } b \equiv 0 \bmod 4\end{cases}
$$

and argue similarly.

Corollary 5. $\alpha\{-n, n, 2 n\}=\alpha\{-1,1,2\}=1 / 3$. 
6.2. Rectangular lattice case. By the rectangular lattice case, we mean that the lattice $\mathcal{K}$ can be generated by vectors $P=(0, p)$ and $Q=(q, 0)$ for suitable choices of $p, q$. Of course, $p$ will necessarily be the $\operatorname{gcd}\left(n_{2}, n_{3}\right) / n_{3}$. In the rectangular lattice case we also have the complete solution to the Kronecker problem.

First, we determine how this situation can arise.

Lemma 9. Suppose $1 \leq n_{1}<n_{2}<n_{3}$ and $\operatorname{gcd}\left(n_{1}, n_{2}, n_{3}\right)=1$. Set $m_{1}=$ $\operatorname{gcd}\left(n_{1}, n_{3}\right)$ and $m_{2}=\operatorname{gcd}\left(n_{2}, n_{3}\right)$. We can choose $P=\left(0, \operatorname{gcd}\left(n_{2}, n_{3}\right) / n_{3}\right)$ if and only if $n_{3}=m_{1} m_{2}$.

Proof. First, we will prove that $C \equiv n_{3} / m_{1} m_{2}$ is an integer and that the pairs $\left(m_{1}, m_{2}\right),\left(m_{1}, n_{2}\right),\left(m_{2}, n_{1}\right),\left(n_{1} / m_{1}, C\right)$ and $\left(n_{2} / m_{2}, C\right)$ are all coprime. To see this, note that if $s$ divides $m_{1}, n_{2}$, then $s$ divides all three integers $n_{1}, n_{2}, n_{3}$ and this is a contradiction. Thus $m_{1}, n_{2}$ are coprime. The argument is the same for the pairs $m_{2}, n_{1}$ and $m_{1}, m_{2}$.

Because $m_{1}$ divides $n_{3}$ and is coprime with $m_{2}$, but $n_{3}=m_{2}\left(n_{3} / m_{2}\right)$, it follows that $m_{1}$ divides $n_{3} / m_{2}$, proving that $n_{3}=m_{1} m_{2} C$ for some integer $C$.

Put $A=n_{1} / m_{1}$. It must be that $A, C$ are coprime, for otherwise $m_{1}$ would not the greatest common divisor of $n_{1}, n_{3}$. Similarly, if $B=n_{2} / m_{2}$, the same reasoning proves $B, C$ are coprime.

Now suppose $P=\left(0, P_{2}\right)$. As $P_{2}=m_{2} / m_{3}$, there is some integer $k$ such that $k n_{2} \equiv m_{2} \bmod n_{3}$ and $k n_{1} \equiv 0 \bmod n_{3}$. Thus there is an integer $j$ such that $k n_{1}=j n_{3}$ which implies that $k A=j C m_{2}$. Since $\operatorname{gcd}(A, C)=1, C$ divides $k$. There is also an integer $J$ such that $k n_{2}+J n_{3}=m_{2}$ and this implies $k B+J m_{1} C=1$. As $C$ divides $k$ we can conclude that $C=1$, i.e., $n_{3}=m_{1} m_{2}$.

Conversely, suppose $n_{3}=m_{1} m_{2}$. As $m_{2}=\operatorname{gcd}\left(n_{2}, n_{3}\right)$, there is some integer $k$ with $k n_{2} \equiv m_{2} \bmod n_{3}$. Because $m_{1} n_{2} \equiv 0 \bmod n_{3}$, for any integer $s$ we have $\left(k-s m_{1}\right) n_{2} \equiv m_{2} \bmod n_{3}$. Since $m_{2}$ and $n_{1}$ are coprime, there are integers $s, t$ such that $k A=m_{2} t+n_{1} s$. It follows that

$$
\frac{\left(k-m_{1} s\right) n_{1}}{n_{3}}=\frac{\left(k-m_{1} s\right) A}{m_{2}}=t .
$$

Therefore $\left(k-m_{1} s\right) n_{1} \equiv 0 \bmod n_{3}$ and so we may take $P=\left(0, m_{2} / n_{3}\right)$.

Theorem 5. Suppose $1 \leq n_{1}<n_{2}<n_{3}$ and $\operatorname{gcd}\left(n_{1}, n_{2}, n_{3}\right)=1$. Set $m_{j}=$ $\operatorname{gcd}\left(n_{j}, n_{3}\right)$ for $j=1,2$ and assume that $n_{3}=m_{1} m_{2}$. Then

and

$$
\begin{aligned}
& \alpha\left\{n_{1}, n_{2}, n_{3}\right\}=\frac{1}{2\left(\frac{n_{1}}{m_{1}}+m_{2}\right)} \text { if } \frac{n_{1}}{m_{1}}+m_{2} \leq m_{1}, \\
& \alpha\left\{n_{1}, n_{2}, n_{3}\right\}=\frac{1}{2\left(\frac{n_{2}}{m_{2}}+m_{1}\right)} \text { if } \frac{n_{2}}{m_{2}}+m_{1} \leq m_{2},
\end{aligned}
$$

$$
\alpha\left\{n_{1}, n_{2}, n_{3}\right\}=\frac{\frac{n_{1}}{m_{1}}+\frac{n_{2}}{m_{2}}}{2\left(n_{1}+n_{2}+\frac{n_{1}}{m_{1}} \frac{n_{2}}{m_{2}}\right)} \text { otherwise. }
$$

Corollary 6. In the rectangular lattice case, $\alpha\left\{n_{1}, n_{2}, n_{3}\right\} \leq 1 / 5$.

Proof. As $m_{1}, m_{2} \geq 2$ and $n_{1} / m_{1}, n_{2} / m_{2} \geq 1$, the claim is obvious if $\alpha$ is given by either of the first two choices. Otherwise note that the inequality

$$
n_{1} m_{2}+n_{2} m_{1} \leq \frac{2}{5}\left(n_{1} m_{1} m_{2}+n_{2} m_{1} m_{2}+n_{1} n_{2}\right)
$$


holds since $m_{j} \geq 2$ and $n_{j} \geq m_{j}$, and this implies that $\alpha \leq 1 / 5$ in the third case.

Of course, a special case of this is the product set $\left\{n_{1}, n_{2}, n_{1} n_{2}\right\}$ when $n_{1} / m_{1}=$ $n_{2} / m_{2}=1$. In this case, the theorem gives the following result.

Corollary 7. Let $1<n_{1}<n_{2}<n_{1} n_{2}$ and suppose $\operatorname{gcd}\left(n_{1}, n_{2}, n_{1} n_{2}\right)=1$. Then

$$
\alpha\left\{n_{1}, n_{2}, n_{1} n_{2}\right\}=\frac{1}{2\left(n_{1}+1\right)} .
$$

Remark 9. It is interesting that $\alpha\left\{n_{1}, n_{2}, n_{1} n_{2}\right\}$ coincides with the trivial lower bound, $\alpha\left\{n_{2}, n_{1} n_{2}\right\}$, and with the upper bound found by geometric methods in Example 6 .

We will make use of the following trivial observation.

Lemma 10. (Same sign lemma) Suppose that $\left|n_{d}\right| \geq\left|n_{k}\right|$ for all $k$. If $u \in \mathbb{R}^{d}$ with $n_{i} u_{i} n_{j} u_{j} \geq 0$ for some $1 \leq i<j \leq d-1$, then

$$
\frac{\left|n_{j} u_{i}-n_{i} u_{j}\right|}{\left|n_{i}\right|+\left|n_{j}\right|} \leq \max \left\{\frac{\left|n_{d} u_{i}\right|}{\left|n_{d}\right|+\left|n_{i}\right|}, \frac{\left|n_{d} u_{j}\right|}{\left|n_{d}\right|+\left|n_{j}\right|}\right\} \text {. }
$$

Proof. The sign condition implies that

$$
\frac{\left|n_{j} u_{i}-n_{i} u_{j}\right|}{\left|n_{i}\right|+\left|n_{j}\right|} \leq \max \left\{\frac{\left|n_{j} u_{i}\right|}{\left|n_{i}\right|+\left|n_{j}\right|}, \frac{\left|n_{i} u_{j}\right|}{\left|n_{i}\right|+\left|n_{j}\right|}\right\} .
$$

Furthermore, if $\left|n_{d}\right| \geq\left|n_{r}\right|$, then $\left|n_{r}\right| /\left(\left|n_{s}\right|+\left|n_{r}\right|\right) \leq\left|n_{d}\right| /\left(\left|n_{s}\right|+\left|n_{d}\right|\right)$.

Proof of Theorem 5. According to the first lemma this is the rectangular lattice case and we can choose $P=\left(0, m_{2} / n_{3}\right)=\left(0,1 / m_{1}\right)$ and $Q=\left(1 / m_{2}, 0\right)$. Put $A=n_{1} / m_{1}$ and $B=n_{2} / m_{2}$. The angular Kronecker constant is the least constant $E$ such that for each $x, y$ there are integers $r, s$ such that

$$
\begin{aligned}
& (2,3): \frac{m_{1} m_{2}}{n_{2}+n_{3}}\left|\frac{r}{m_{1}}-y\right|=\frac{\left|r-m_{1} y\right|}{B+m_{1}} \leq E, \\
& (1,3): \frac{m_{1} m_{2}}{n_{1}+n_{3}}\left|\frac{s}{m_{2}}-x\right|=\frac{\left|s-m_{2} x\right|}{A+m_{2}} \leq E,
\end{aligned}
$$

and

$$
(1,2): \frac{\left|n_{2}\left(\frac{s}{m_{2}}-x\right)-n_{1}\left(\frac{r}{m_{1}}-y\right)\right|}{n_{1}+n_{2}}=\frac{\left|B\left(s-m_{2} x\right)-A\left(r-m_{1} y\right)\right|}{n_{1}+n_{2}} \leq E .
$$

We present upper bound arguments first. This proceeds by the cases described in the theorem.

First, suppose $B+m_{1} \leq m_{2}$. Then also $m_{1} \leq A+B+m_{2}$, so if we put

$$
\delta:=\frac{A\left(A+B+m_{2}-m_{1}\right)}{2\left(n_{1}+2 n_{2}+A B\right)},
$$

then $\delta \geq 0$. Moreover, this choice of $\delta$ gives

$$
\frac{\frac{1}{2}+\delta}{A+m_{2}}=\frac{\frac{A+B}{2}-B \delta}{n_{1}+n_{2}}
$$

Pick $r$ such that $\left|r-m_{1} y\right| \leq 1 / 2$, so

$$
(2,3) \leq \frac{1}{2\left(B+m_{1}\right)}
$$


Suppose, first, there is an integer $s^{\prime}$ such that $\left|s^{\prime}-m_{2} x-1 / 2\right| \leq \delta_{0}$, where $\delta_{0}=$ $\min (\delta, 1 / 2)$. Then the triangle inequality shows that both $\left|s^{\prime}-m_{2} x\right| \leq \delta_{0}+1 / 2$ and $\left|s^{\prime}-m_{2} x-1\right| \leq \delta_{0}+1 / 2$. Moreover, $s^{\prime}-1 \leq m_{2} x \leq s^{\prime}$. Taking $s=s^{\prime}$ or $s^{\prime}-1$, appropriately, we can arrange for $\operatorname{sign}\left(s-m_{2} x\right)=\operatorname{sign}\left(r-m_{1} y\right)$ and $\left|s-m_{2} x\right| \leq \delta+1 / 2$. Then the definition of $\delta$ and the fact that $B+m_{1} \leq m_{2}$ yields,

$$
(1,3) \leq \frac{\frac{1}{2}+\delta}{A+m_{2}}=\frac{2 B+A}{2\left(n_{1}+2 n_{2}+A B\right)} \leq \frac{1}{2\left(B+m_{1}\right)} .
$$

By the same sign lemma, $(1,2) \leq 1 /\left(2 B+2 m_{1}\right)$.

Otherwise, there is no integer $s^{\prime}$ in the interval $\left[m_{2} x+1 / 2-\delta, m_{2} x+1 / 2+\delta\right]$ (and, necessarily, $\delta \leq 1 / 2$ ). Of course there is an integer $s^{\prime}$ in the interval $\left[m_{2} x+\right.$ $\left.1 / 2+\delta-1, m_{2} x+1 / 2+\delta\right]$ and hence in $\left[m_{2} x-1 / 2+\delta, m_{2} x+1 / 2-\delta\right)$. So there is an integer $s$ such that so that $\left|s-m_{2} x\right| \leq 1 / 2-\delta$. As above, the definition of $\delta$ gives

$$
(1,3) \leq \frac{\frac{1}{2}-\delta}{A+m_{2}}<\frac{1}{2\left(B+m_{1}\right)}
$$

and

$$
\begin{gathered}
(1,2) \leq \frac{B\left(\frac{1}{2}-\delta\right)}{n_{1}+n_{2}}+\frac{A}{2\left(n_{1}+n_{2}\right)}=\frac{\frac{A+B}{2}-B \delta}{n_{1}+n_{2}} \\
=\frac{\frac{1}{2}+\delta}{A+m_{2}} \leq \frac{1}{2\left(B+m_{1}\right)} .
\end{gathered}
$$

Thus in either case, each of the three inequalities is bounded by $\frac{1}{2\left(B+m_{1}\right)}$, as claimed.

The case $A+m_{2} \leq m_{1}$ is similar.

The final upper bound case to consider is $B+m_{1}>m_{2}$ and $A+m_{2}>m_{1}$. Put

$$
\delta_{y}:=\frac{B\left(B+m_{1}-m_{2}\right)}{2\left(n_{1}+n_{2}+A B\right)}
$$

and

$$
\delta_{x}:=\left(\frac{1}{2}+\delta_{y}\right) \frac{A+m_{2}}{B+m_{1}}-\frac{1}{2}=\frac{A\left(A+m_{2}-m_{1}\right)}{2\left(n_{1}+n_{2}+A B\right)} .
$$

Both $\delta_{x}, \delta_{y}>0$. They were chosen so

$$
\frac{\frac{1}{2}+\delta_{y}}{B+m_{1}}=\frac{\frac{1}{2}+\delta_{x}}{A+m_{2}}=\frac{B\left(\frac{1}{2}-\delta_{x}\right)+A\left(\frac{1}{2}-\delta_{y}\right)}{n_{1}+n_{2}} .
$$

Furthermore, these three (equal) expressions coincide with

$$
E=\frac{A+B}{2\left(n_{1}+n_{2}+A B\right)} .
$$

We prove next that $\delta_{y}<1 / 2$. Because $m_{2} B=n_{2}<n_{3}=m_{2} m_{1}$ we have $B<m_{1}$. Thus

$$
\begin{aligned}
B\left(B+m_{1}-m_{2}\right) & =B\left(B+m_{1}\right)-n_{2}<2 B m_{1}-n_{2} \\
& <2 B\left(A+m_{2}\right)-n_{2}=A B+n_{2}+A B \\
& <A m_{1}+n_{2}+A B=n_{1}+n_{2}+A B
\end{aligned}
$$

Likewise, $\delta_{x}<1 / 2$. 
Suppose there is an integer $s^{\prime}$ such that $\left|s^{\prime}-m_{2} x-1 / 2\right| \leq \delta_{x}$. Pick an integer $r$ such that $\left|r-m_{1} y\right| \leq 1 / 2$ and choose the integer $s$ such that $\left|s-m_{2} x\right| \leq 1 / 2+\delta_{x}$ and $\operatorname{sign}\left(s-m_{2} x\right)=\operatorname{sign}\left(r-m_{1} y\right)$. Then

$$
(1,3) \leq \frac{\frac{1}{2}+\delta_{x}}{A+m_{2}}=\frac{A+B}{2\left(n_{1}+n_{2}+A B\right)}
$$

and

$$
(2,3) \leq \frac{1}{2\left(B+m_{1}\right)} \leq \frac{\frac{1}{2}+\delta_{y}}{B+m_{1}}=\frac{A+B}{2\left(n_{1}+n_{2}+A B\right)} .
$$

By the same sign lemma we also have

$$
(1,2) \leq \frac{A+B}{2\left(n_{1}+n_{2}+A B\right)} .
$$

Otherwise, there is an integer $s$ such that $\left|s-m_{2} x\right| \leq 1 / 2-\delta_{x}$. If there is some integer $r^{\prime}$ such that $\left|r^{\prime}-m_{1} y-1 / 2\right| \leq \delta_{y}$, then we can choose $r$ such that $\left|r-m_{1} y\right| \leq 1 / 2+\delta_{y}$ and $\operatorname{sign}\left(s-m_{2} x\right)=\operatorname{sign}\left(r-m_{1} y\right)$. Similar arguments to those above show that $(1,2),(1,3)$ and $(2,3)$ are all dominated by

$$
\frac{A+B}{2\left(n_{1}+n_{2}+A B\right)} \text {. }
$$

Otherwise, there are integers $r$ and $s$ with $\left|r-m_{1} y\right| \leq 1 / 2-\delta_{y}$ and $\left|s-m_{2} x\right| \leq$ $1 / 2-\delta_{x}$. Then

$$
(1,3) \leq \frac{\frac{1}{2}-\delta_{x}}{A+m_{2}}, \quad(2,3) \leq \frac{\frac{1}{2}-\delta_{y}}{B+m_{1}}
$$

and by triangle inequality,

$$
(1,2) \leq \frac{B\left(\frac{1}{2}-\delta_{x}\right)+A\left(\frac{1}{2}-\delta_{y}\right)}{n_{1}+n_{2}} .
$$

Hence, again, all three are dominated by

$$
\frac{A+B}{2\left(n_{1}+n_{2}+A B\right)} \text {. }
$$

This completes the proof that $E$ is an upper bound for the angular Kronecker constant.

We now turn to proving that the claimed values are lower bounds for the angular Kronecker constant. Since the angular Kronecker constant of $\left\{A m_{1}, B m_{2}, m_{1} m_{2}\right\}$ must be at least the angular Kronecker constants of both of the two element sets, $\left\{A m_{1}, m_{1} m_{2}\right\}$ and $\left\{B m_{2}, m_{1} m_{2}\right\}$, it must be at least the greater of $1 / 2\left(A+m_{2}\right)$ and $1 / 2\left(B+m_{1}\right)$. Thus we only need to verify the sharpness of the choice of $E$ for the case $B+m_{1}>m_{2}$ and $A+m_{2}>m_{1}$. Take

$$
x=\frac{(A+B)\left(A+m_{2}\right)}{2 m_{2}\left(A B+n_{1}+n_{2}\right)}
$$

and

$$
y=\frac{n_{1}+n_{2}+A B-B\left(B+m_{1}-m_{2}\right)}{2 m_{1}\left(A B+n_{1}+n_{2}\right)} .
$$

We will show that for any choice of integers $r, s$

$$
\max \left(\frac{\left|r-m_{1} y\right|}{B+m_{1}}, \frac{\left|s-m_{2} x\right|}{A+m_{2}}, \frac{\left|B\left(s-m_{2} x\right)-A\left(r-m_{1} y\right)\right|}{n_{1}+n_{2}}\right) \geq \frac{A+B}{2\left(n_{1}+n_{2}+A B\right)} .
$$


Let $\delta_{x}, \delta_{y}$ be as defined in $(4,5)$. Then $m_{2} x=1 / 2+\delta_{x}$ and $m_{1} y=1 / 2-\delta_{y}$. Because $0<\delta_{x}, \delta_{y}<1 / 2, m_{2} x, m_{1} y \in(0,1)$.

Furthermore,

$$
\begin{aligned}
\frac{(A+B)}{2\left(n_{1}+n_{2}+A B\right)} & \left.=\frac{1 / 2+\delta_{y}}{B+m_{1}}=\frac{\left|1-m_{1} y\right|}{B+m_{1}} \quad \text { (Condition }(2,3) \text { with } r=1\right) \\
& =\frac{1 / 2+\delta_{x}}{A+m_{2}}=\frac{\left|m_{2} x\right|}{A+m_{2}} \quad(\text { Condition }(1,3) \text { with } s=0) \\
& =\frac{\left|B\left(1 / 2-\delta_{x}\right)+A\left(1 / 2-\delta_{y}\right)\right|}{n_{1}+n_{2}} \\
& \left.=\frac{\left|B\left(1-m_{2} x\right)+A m_{1} y\right|}{n_{1}+n_{2}} \quad \text { (Condition }(1,2) \text { with } r=0, s=1\right) .
\end{aligned}
$$

Since $m_{2} x, m_{1} y \in(0,1)$

$$
\begin{aligned}
& \frac{\left|r-m_{1} y\right|}{B+m_{1}} \geq \frac{\left|1-m_{1} y\right|}{B+m_{1}} \text { if } r \geq 1 \text { or } r \leq-1 \\
& \frac{\left|s-m_{2} x\right|}{A+m_{2}} \geq \frac{\left|m_{2} x\right|}{A+m_{2}} \text { if } s \leq 0 \text { or } s \geq 2
\end{aligned}
$$

This exhausts all possibilities for integers $r, s$ and completes the proof that $E$ is sharp.

6.3. Generalized sum sets. It was shown in [Graham and Hare 2006a, Prop. 2.6] that $\alpha\left\{n_{1}, n_{2}, n_{1}+n_{2}\right\} \geq 1 / 6$. Using Theorem 4 the Kronecker constant for $\left\{n_{1}, n_{2}, n_{1}+n_{2}\right\}$ can be exactly determined and it is asymptotically $1 / 6$.

Theorem 6. Let $1 \leq n_{1}<n_{2}<n_{3}$ with $n_{3}=n_{1}+n_{2}$ and $\operatorname{gcd}\left(n_{1}, n_{2}, n_{3}\right)=1$. Suppose $n_{1}+2 n_{2} \equiv j \bmod 3$. Then

$$
\begin{aligned}
\alpha\left\{n_{1}, n_{2}, n_{3}\right\} & =\frac{1}{6}+\frac{1}{2\left(n_{1}+2 n_{2}\right)} \text { if } j=0 ; \\
\alpha\left\{n_{1}, n_{2}, n_{3}\right\} & =\frac{1}{6}+\frac{1}{3\left(n_{1}+2 n_{2}\right)} \text { if } j=1 \text {; and } \\
\alpha\left\{n_{1}, n_{2}, n_{3}\right\} & =\frac{1}{6}+\frac{1}{3\left(2 n_{1}+n_{2}\right)} \text { if } j=2 .
\end{aligned}
$$

Corollary 8. $\alpha\{1,2,3\}=1 / 4 ; \alpha\left\{n_{1}, n_{2}, n_{1}+n_{2}\right\} \leq 1 / 4$ for all $0<n_{1}<n_{2}<$ $n_{1}+n_{2}$.

Proof. We remark that the condition $\operatorname{gcd}\left(n_{1}, n_{2}, n_{3}\right)=1 \operatorname{implies} \operatorname{gcd}\left(n_{2}, n_{3}\right)=1$. Thus $Q=(1,0)$ and since $t n_{2} \equiv-t n_{1} \bmod \left(n_{1}+n_{2}\right)$ we can take $P=\left(-1 / n_{3}, 1 / n_{3}\right)$. Hence the angular Kronecker constant is the least $E$ such that for all $x, y$ there are integers $s, t$ such that the following three inequalities are satisfied:

$$
\begin{aligned}
(2,3) & : \quad \frac{n_{3}}{n_{2}+n_{3}}\left|\frac{t}{n_{3}}-y\right| \leq E \\
(1,3) & : \quad \frac{n_{3}}{n_{1}+n_{3}}\left|-\frac{t}{n_{3}}+s-x\right| \leq E \\
(1,2): & : \frac{\left|n_{2}\left(-\frac{t}{n_{3}}+s-x\right)-n_{1}\left(\frac{t}{n_{3}}-y\right)\right|}{n_{1}+n_{2}} \leq E .
\end{aligned}
$$


Setting $\Delta_{t}=t / n_{3}-y$ and letting $\beta \in[0,1]$ be congruent to $x+y \bmod 1$, the three inequalities can be rewritten with an integer $s^{\prime}$ as

$$
\begin{array}{ll}
(2,3)^{\prime}: & \left|\Delta_{t}\right| \leq E\left(\frac{n_{2}+n_{3}}{n_{3}}\right)=E\left(\frac{n_{2}}{n_{1}+n_{2}}+1\right) \\
(1,3)^{\prime}: & \left|\Delta_{t}+\beta+s^{\prime}\right| \leq E\left(\frac{n_{1}}{n_{1}+n_{2}}+1\right) \\
(1,2)^{\prime}: & \left|\Delta_{t}\left(1+\frac{n_{1}}{n_{2}}\right)+\beta+s^{\prime}\right| \leq E\left(\frac{n_{1}}{n_{2}}+1\right) .
\end{array}
$$

Upper bound argument: Clearly, $(2,3)^{\prime}$ is satisfied if

$$
\Delta_{t} \in I_{1}=\left[-E\left(\frac{n_{2}}{n_{1}+n_{2}}+1\right), E\left(\frac{n_{2}}{n_{1}+n_{2}}+1\right)\right],
$$

With $s^{\prime}=0,-1,(1,3)^{\prime}$ is satisfied precisely when $\Delta_{t} \in I_{2} \cup I_{2}^{\prime}$, where

$$
\begin{aligned}
& I_{2}=\left[-E\left(\frac{n_{1}}{n_{1}+n_{2}}+1\right)-\beta, E\left(\frac{n_{1}}{n_{1}+n_{2}}+1\right)-\beta\right], \\
& I_{2}^{\prime}=\left[-E\left(\frac{n_{1}}{n_{1}+n_{2}}+1\right)+1-\beta, E\left(\frac{n_{1}}{n_{1}+n_{2}}+1\right)+1-\beta\right] .
\end{aligned}
$$

Similarly, $(1,2)^{\prime}$ is satisfied for $s^{\prime}=0,-1$ when $\Delta_{t} \in I_{3} \cup I_{3}^{\prime}$, where

$$
\begin{aligned}
& I_{3}=\left[-E-\beta \frac{n_{2}}{n_{1}+n_{2}}, E-\beta \frac{n_{2}}{n_{1}+n_{2}}\right], \\
& I_{3}^{\prime}=\left[-E+(1-\beta) \frac{n_{2}}{n_{1}+n_{2}}, E+(1-\beta) \frac{n_{2}}{n_{1}+n_{2}}\right] .
\end{aligned}
$$

If we let $I_{i}=\left[A_{i}, B_{i}\right]$, then

$$
\begin{aligned}
& A_{3}-A_{2}=\frac{(\beta+E) n_{1}}{n_{1}+n_{2}}, A_{1}-A_{3}=\frac{(\beta-E) n_{2}}{n_{1}+n_{2}}, \\
& B_{1}-B_{3}=\frac{(\beta+E) n_{2}}{n_{1}+n_{2}}, \quad B_{2}-B_{3}=\frac{(E-\beta) n_{1}}{n_{1}+n_{2}} .
\end{aligned}
$$

The reader can check from these facts that the interval $I_{1} \cap I_{2} \bigcap I_{3}$ (the case $s^{\prime}=0$ ) equals

and

$$
J_{1}:=\left[-E-\beta \frac{n_{2}}{n_{1}+n_{2}}, E-\beta \frac{n_{2}}{n_{1}+n_{2}}\right] \text { if } \beta \leq E
$$

$$
J_{2}:=\left[-E\left(\frac{n_{2}}{n_{1}+n_{2}}+1\right), E\left(\frac{n_{1}}{n_{1}+n_{2}}+1\right)-\beta\right] \text { if } \beta \geq E .
$$

Similarly, one can show that the interval $I_{1} \cap I_{2}^{\prime} \cap I_{3}^{\prime}$ (the case $s^{\prime}=-1$ ) equals

$$
J_{2}^{\prime}:=\left[-E\left(\frac{n_{1}}{n_{1}+n_{2}}+1\right)+1-\beta, E\left(\frac{n_{2}}{n_{1}+n_{2}}+1\right)\right] \text { if } \beta \leq 1-E
$$

and

$$
J_{1}^{\prime}:=\left[-E+(1-\beta) \frac{n_{2}}{n_{1}+n_{2}}, E+(1-\beta) \frac{n_{2}}{n_{1}+n_{2}}\right] \text { if } \beta \geq 1-E .
$$

For $\beta \leq E$, we want to show that for all $y$ we can choose an integer $t$ such that $\Delta_{t}=t / n_{3}-y \in J_{1}$. As this interval has width $2 E \geq 1 / 3 \geq 1 / n_{3}$, this can always be done. A similar argument applies with $J_{1}^{\prime}$ if $\beta \geq 1-E$.

For $E \leq \beta \leq 1-E$, it is enough to show that we can choose $\Delta_{t}$ in $J_{2}$ or $J_{2}^{\prime}$. To do this we will prove that sum of the lengths of those two intervals is at least $1 / n_{3}$ 
and that the left endpoint of one is congruent $\left(\bmod 1 / n_{3}\right)$ to the right endpoint of the other. These two properties are clearly enough to ensure there is a choice of $\Delta_{t} \in J_{2} \cup J_{2}^{\prime}$.

The combined widths of these two intervals is

$$
2 E\left(\frac{2 n_{2}+n_{1}}{n_{1}+n_{2}}\right)+2 E\left(\frac{2 n_{1}+n_{2}}{n_{1}+n_{2}}\right)-1=6 E-1
$$

and this certainly exceeds $1 / n_{3}$ for the specified values of $E$.

It is in checking that the left endpoint of one interval is congruent to the right endpoint of the other that the dependence of $E$ on the congruence of $n_{1}+2 n_{2}$ is relevant. We want to verify that either

$$
2 E\left(1+\frac{n_{2}}{n_{3}}\right) \in \frac{\mathbb{Z}}{n_{3}}
$$

or

$$
2 E\left(1+\frac{n_{1}}{n_{3}}\right)-1 \in \frac{\mathbb{Z}}{n_{3}} .
$$

Indeed, the first condition holds if $n_{1}+2 n_{2}$ is either congruent to 0 or $1 \bmod 3$. Using the fact that $2 n_{1}+n_{2} \equiv-\left(n_{1}+2 n_{2}\right) \bmod n_{3}$, one can see that the second condition is satisfied when $n_{1}+2 n_{2} \equiv 2 \bmod 3$.

This completes the argument that $E$ is an upper bound on the Kronecker constant.

Lower bound: Now suppose some $E_{\epsilon}<E$ can play the role of $E$ in $(1,2)^{\prime},(1,3)^{\prime}$ and $(2,3)^{\prime}$ for all $x, y$. Since $E \leq 1 / 4$, condition $(2,3)^{\prime}$ implies that

$$
\left|\Delta_{t}\right| \leq E_{\varepsilon}\left(\frac{n_{2}+n_{3}}{n_{3}}\right)<1 / 2
$$

Let $\beta=1 / 2$. Together with $(1,3)^{\prime}$ and $(2,3)^{\prime}$, this implies that $s^{\prime}$ can only take on the values 0 or -1 . With $\beta=1 / 2, \Delta_{t}$ must be contained in the interior of the disjoint, symmetric intervals, $J_{2}$ and $J_{2}^{\prime}$. The sum of the lengths of these two intervals is clearly less than $2 / n_{3}$, so each interval, $J_{2}, J_{2}^{\prime}$, has length less than $1 / n_{3}$.

If $n_{1}+2 n_{2} \equiv 0 \bmod 3$, say $n_{1}+2 n_{2}=3 L$, then the left-hand endpoint of $J_{2}$ is

$$
-\left(\frac{1}{6}+\frac{1}{2\left(n_{1}+2 n_{2}\right)}\right)\left(\frac{2 n_{2}+n_{1}}{n_{1}+n_{2}}\right)=-\frac{L+1}{2 n_{3}},
$$

so the interior of $J_{2}$ is contained in

$$
\left(\frac{-(L+1)}{2 n_{3}}, \frac{-L+1}{2 n_{3}}\right) .
$$

$J_{2}^{\prime}$ is similar. If $L$ is odd, take $y=0, x=1 / 2$. If $L$ is even, take $y=1 /\left(2 n_{3}\right)$, $x=1 / 2-y$. With these choices for $y$, there is no integer $t$ such that $\Delta_{t}=t / n_{3}-y \in$ $\operatorname{int}\left(J_{2} \cup J_{2}^{\prime}\right)$, as is necessary for all three of $(1,2)^{\prime},(1,3)^{\prime}$ and $(2,3)^{\prime}$ to hold with $E_{\epsilon}$ in the place of $E$. If $n_{1}+2 n_{2} \equiv 1 \bmod 3$ the argument is similar.

Lastly, if $n_{1}+2 n_{2} \equiv 2 \bmod 3$, say $n_{1}+2 n_{2}=3 L+2$, then since $2 n_{1}+n_{2}=$ $3 n_{3}-\left(n_{1}+2 n_{2}\right)$, a calculation similar to the one above shows the right-hand endpoint of $J_{2}$ is $-L /\left(2 n_{3}\right)$. Again, taking $y=0$ and $x=1 / 2$ if $L$ is even, or $y=1 /\left(2 n_{3}\right)$ and $x=1 / 2-y$ if $L$ is odd, the rest of the argument for this case proceeds like that for the case of $n_{1}+2 n_{2} \bmod 1$ being 0 .

This completes the proof that $E$ is the Kronecker constant. 
Using similar arguments we have been able to compute the exact Kronecker constants for arithmetic progressions, as well. This is a special case of the following theorem on generalized arithmetic progressions whose proof is omitted.

Theorem 7. Suppose $1 \leq n_{1}<n_{2}<n_{3}$ and $\operatorname{gcd}\left(n_{1}, n_{2}, n_{3}\right)=1$. Assume that there is a positive integer $r$ such that $r n_{2}=(r-1) n_{3}+n_{1}$ and suppose that $n_{2} \geq$ $2 r(r-1)$. Assume $n_{2}+n_{3} \equiv j \bmod 2 r$, with $j \in\{0,1, \ldots, 2 r-1\}$. Then $\alpha\left\{n_{1}, n_{2}, n_{3}\right\}$ equals

$$
\begin{gathered}
\frac{1}{4 r}+\frac{1}{2\left(n_{2}+n_{3}\right)}-\frac{j}{4 r\left(n_{2}+n_{3}\right)} \text { if } j=0,1 ; \\
\frac{1}{4 r}+\frac{j(r-1)}{4 r\left(n_{1}+n_{2}\right)} \text { if }\left(j=3 \text { and } 2 n_{1} \geq n_{2}\right) \text { or } j=2 ;
\end{gathered}
$$

and

$$
\frac{1}{4 r}+\frac{1}{n_{2}+n_{3}}-\frac{j}{4 r\left(n_{2}+n_{3}\right)} \text { if }\left(j=3 \text { and } 2 n_{1} \leq n_{2}\right) \text { or } j \geq 4 .
$$

In particular,

$$
\frac{1}{4 r} \leq \alpha\left\{n_{1}, n_{2}, n_{3}\right\} \leq \frac{1}{4 r}+\frac{1}{n_{2}+n_{3}} .
$$

Corollary 9. Suppose $1 \leq n_{1}<n_{2}<n_{3}$ is an arithmetic progression with $n_{2} \geq 4$ and $\operatorname{gcd}\left(n_{1}, n_{2}, n_{3}\right)=1$. If $n_{2}+n_{3} \equiv j \bmod 4$ and $n_{j+1}-n_{j}=d$, the angular Kronecker constant, $\alpha\left\{n_{1}, n_{2}, n_{3}\right\}$ is given by the formula

$$
\begin{array}{ll}
\frac{1}{8}+\frac{1}{2\left(2 n_{1}+3 d\right)}-\frac{j}{8\left(2 n_{1}+3 d\right)} & \text { if } j=0,1, \\
\frac{1}{8}+\frac{j}{8\left(2 n_{1}+d\right)} & \text { if }\left(j=3 \text { and } 2 n_{1} \geq n_{2}\right) \text { or } j=2, \\
\frac{1}{8}+\frac{1}{2 n_{1}+3 d}-\frac{3}{8\left(2 n_{1}+3 d\right)} & \text { if } j=3 \text { and } 2 n_{1} \leq n_{2} .
\end{array}
$$

Proof. Arithmetic progressions with no common divisor and $n_{2} \geq 4$ are the special case of the theorem with $r=2$.

Remark 10. Note that $j \equiv-d \bmod 4$ when $n_{3}$ is even and $j \equiv 2-d \bmod 4$ when $n_{3}$ is odd. In particular, when the step size $d=1$, we have $j=1$ or 3 and the assumption $n_{2} \geq 4$ implies $2 n_{1}>n_{2}$. Consequently, the conclusion of Corollary 9 yields:

$$
\alpha\left\{n_{1}, n_{1}+1, n_{1}+2\right\}=\frac{\left\lceil n_{1} / 2\right\rceil+1}{\left(8\left\lceil n_{1} / 2\right\rceil+2\right)} \text { whenever } n_{1} \geq 3 .
$$

This improves the observation in [Graham and Hare 2006a, Prop. 2.6] that $\kappa\left\{n_{1}, n_{1}+\right.$ $\left.1, n_{1}+2\right\} \geq|1-\exp i \pi / 4|$.

Of course, if $\left\{n_{1}, n_{2}, n_{3}\right\}$ is an arithmetic progression with common divisor $q$, then the angular Kronecker constant of $\left\{n_{1}, n_{2}, n_{3}\right\}$ is the same as the angular Kronecker constant of the arithmetic progression $\left\{n_{1} / q, n_{2} / q, n_{3} / q\right\}$.

Remark 11. The requirement in the corollary that $n_{2} \geq 4$ is a sufficient, but not necessary condition. There are three arithmetic progressions (with no common divisor) which do not satisfy this condition: $\{1,2,3\},\{2,3,4\}$ and $\{1,3,5\}$. For the latter two the Kronecker constants are $1 / 5$ and $3 / 16$ (determined by computer algorithm) respectively, agreeing with the formula. However, we have already seen that $\alpha\{1,2,3\}=1 / 4$, larger than the value $2 / 9$ given by the formula above. 
Remark 12. Another interesting example is $\{5,11,13\}$. This is a generalized arithmetic progression with $r=4$. It fails to satisfy the hypothesis in the theorem that $n_{2} \geq 2 r(r-1)$. Numerical work shows that the Kronecker constant is 59/526, which is greater than the formula value, 1/12.

The sets $\{1, r, r+1\}$ are also generalized arithmetic progressions since $r \cdot r=$ $(r-1)(r+1)+1$ and, of course, the condition $n_{2} \geq 2 r(r-1)$ fails to hold. As $n_{2}+n_{3} \equiv 1 \bmod 2 r$ the theorem formula simplifies to $\alpha=1 /(2 r+1)$ and this is always strictly smaller than the correct value given for $\alpha\{1, r, r+1\}$ in Theorem 6 .

We will say a set of non-zero integers, $\left\{n_{j}\right\}$, is $k$-independent if whenever $\sum \varepsilon_{j} n_{j}=$ 0 with $\varepsilon_{j} \in\{0, \pm 1, \ldots, \pm k\}$ then $\varepsilon_{j}=0$ for all $j$. If a set is $k$-independent, but not $k+1$-independent, we will call it sharp $k$-independent. A 2 -independent set is sometimes called dissociate and is an example of a Sidon set [Lopez and Ross 1975]. Sum sets are (typically) sharp 0-independent sets; arithmetic progressions are either sharp 0 or 1-independent. Actually, the sharp 0-independent sets of size three are precisely those of the form $\{-a, a, b\}$, which have Kronecker constant at least $1 / 4$, and the sets $\left\{ \pm n_{1}, \pm n_{2}, \pm n_{3}\right\}$ with $n_{1}+n_{2}=n_{3}$. Thus if we let $\beta_{k}=\inf \{\alpha(S): S$ is sharp $k$-independent, $|S|=3\}$, then the sum set theorem implies $\beta_{0}=1 / 6$.

Our numerical work suggests that the Kronecker constant of a set depends on the arithmetic relations it satisfies. Indeed, it is an easy consequence of the lower bound for sum sets that $\beta_{k}$ is bounded away from zero.

Proposition 10. Suppose $S=\left\{n_{1}, n_{2}, n_{3}\right\}$ is a sharp $(k-1)$-independent set. Then $\alpha(S) \geq 1 /(6 k)$.

Proof. As $\alpha\{-a, a, b\} \geq 1 / 4$, and all the sets $\left\{ \pm n_{1}, \pm n_{2}, \pm n_{3}\right\}$ have the same independence properties, we can assume $0<n_{1}<n_{2}<n_{3}$.

Suppose $k_{1} n_{1}+k_{2} n_{2}=k_{3} n_{3}$ with $\left|k_{j}\right| \leq k$ (and some $\left|k_{j}\right|=k$.) If some $k_{j}=0$ then, without loss of generality, $k n_{1}=j n_{2}$ with $j \leq k$. Put $m=\operatorname{gcd}\left(n_{1}, n_{2}\right)$ and suppose $n_{i}=m p_{i}$ for $i=1,2$. Since $k m p_{1}=j m p_{2}$ and $\operatorname{gcd}\left(p_{1}, p_{2}\right)=1$, we must have $p_{1}$ dividing $j$ and $p_{2}$ dividing $k$. Thus $1 \leq p_{1}, p_{2} \leq k$. As the Kronecker constant of the two element set $\left\{n_{1}, n_{2}\right\}$ is a lower bound on $\alpha\left\{n_{1}, n_{2}, n_{3}\right\}$ it follows that $\alpha(S) \geq 1 /\left(2 p_{1}+2 p_{2}\right) \geq 1 /(4 k)$.

Similarly, if some $\left|k_{i} n_{i}\right|=\left|k_{j} n_{j}\right|$, we have $\alpha(S) \geq 1 /(4 k)$.

Hence we can assume all $k_{j} \neq 0$ and all $\left|k_{i} n_{i}\right|$ are distinct. Moreover, by interchanging the $n_{i}$, if necessary, we can assume all $k_{i} n_{i}>0$. Thus by the sum set theorem, $\alpha\left\{k_{1} n_{1}, k_{2} n_{2}, k_{3} n_{3}\right\} \geq 1 / 6$.

Since $\left|k_{j}\right| \cdot\left|n_{j} x-t_{j} / k_{j}\right|=\left|k_{j} n_{j} x-t_{j}\right|, \alpha\left\{k_{1} n_{1}, k_{2} n_{2}, k_{3} n_{3}\right\} \leq k \alpha\left\{n_{3}, n_{1}, n_{2}\right\}$ and therefore $\alpha(S) \geq 1 /(6 k)$.

6.4. Remarks on upper bounds for any three element set. There is an easy upper bound (better than 1/2) for any finite set that does not contain 0 .

Proposition 11. If $S$ is a set of $d$ distinct, non-zero integers then $\alpha(S) \leq 1 / 2-$ $1 /(2 d)$.

Proof. Suppose $f: S \rightarrow \mathbb{R}$ and fix $\varepsilon<1 /(2 d)$. For each $j$ let

$$
A_{j}=\left\{x \in[-1 / 2,1 / 2]:\left|\operatorname{Arg}\left(\exp 2 \pi i\left(f\left(n_{j}\right)-n_{j} x\right)\right)\right| \leq 1 / 2-\varepsilon\right\} .
$$

If for each choice of angles $f$, the set $\bigcap_{j=1}^{d} A_{j}$ is non-empty then $S$ is clearly at least $1 / 2-\varepsilon$-angular Kronecker. 
But $A_{j}$ is the union of $n_{j}$ disjoint intervals whose lengths sum to $1-2 \varepsilon$. Thus the measure of $\bigcup_{j=1}^{d} A_{j}^{c}$ is at most $2 d \varepsilon<1$ and hence $\bigcap_{j=1}^{d} A_{j}$ cannot be empty.

Remark 13. When $d=2$, this gives $\alpha(S) \leq 1 / 4$ and this trivial upper bound is sharp if and only if $S=\{-n, n\}$. For $d=3$, the value is $1 / 3$ and Cor. 5 shows that this is sharp if $S=\{-n, n, 2 n\}$. Using our methods we can also prove that that the angular Kronecker constant is strictly less than $1 / 3$ for all other three element sets (that exclude 0). This is very technical, whose proof is not included here but has been archived at [Hare and Ramsey 2011].

We conjecture that $\alpha\left\{n_{1}, n_{2}, n_{3}\right\} \leq 1 / 4$ for all three element sets other than $\{-n, n, 2 n\}$. We have already seen this is true in the rectangular lattice and the sum set case. As well, we have run our computer algorithm on all three element sets of positive integers with $n_{3} \leq 50$ and the greatest Kronecker constant is $1 / 4$, occurring only on the integer multiples of $\{1,2,3\}$. The archive [Hare and Ramsey 2011] has a table of these Kronecker constants.

\section{Computing Kronecker Constants}

For a set of integers $T$ of size $d$, computing the angular Kronecker constant $\alpha(T)$ is equivalent to performing nested, but opposite optimizations over some infinite sets:

where

$$
\alpha(T)=\max \left\{\alpha_{T}(f): f \in \mathbb{R}^{d-1} \times\{0\}\right\}
$$

$$
\alpha(f)=\alpha_{T}(f)=\min \left\{\|f-k\|_{L}: k \in \mathcal{K}\right\} .
$$

Because there is a finite set $\mathcal{V} \subset \mathbb{R}^{d}$ for which

$$
\|u\|_{L}=\max \{|v \cdot u|: v \in \mathcal{V}\}
$$

the innermost maximization is computable. Moreover, $\mathcal{V}$ depends only on $T$ simply and explicitly, and has size is $d(d-1) / 2$. By replacing $\mathcal{V}$ with $\mathcal{V} \cup(-\mathcal{V})$, the innermost maximization becomes a maximum of linear functions (removing the absolute values in the expression):

$$
\|u\|_{L}=\max \{v \cdot u: v \in \mathcal{V} \cup(-\mathcal{V})\}
$$

Second, dependent only on $T$ simply and explicitly, we select a compact, convex set $\mathcal{D}^{\prime}$, determined by a finite set of linear inequalities $\mathcal{H}$, such that

$$
\alpha(T)=\max \left\{\alpha_{T}(f): f \in \mathcal{D}^{\prime}\right\}
$$

Third, given $\mathcal{D}^{\prime}$, we select a finite $S \subset \mathcal{K}$ such that, for all $f \in \mathcal{D}^{\prime}$,

$$
\min \left\{\|f-k\|_{L}: k \in S\right\}=\min \left\{\|f-k\|_{L}: k \in \mathcal{K}\right\}=\alpha(f)
$$

We then solve the many linear programming problems implied by our choices.

- For every $(k, v) \in S \times[\mathcal{V} \cup(-\mathcal{V})]$, there are linear constraints $\mathcal{N}(k, v)$ that describe a convex set of $f \in \mathbb{R}^{d-1}$ for which $\|f-k\|=(f-k) \cdot v$.

- For every $\tau: S \rightarrow[\mathcal{V} \cup(-\mathcal{V})]$, let

$$
\mathcal{M}(\tau)=\mathcal{H} \cup\left(\bigcup_{k \in \mathcal{S}} \mathcal{N}(k, \tau(k))\right)
$$

- For each $k \in S$, there are linear constraints $\mathcal{J}_{k}$ that express the fact that $\|f-k\|_{L} \leq\left\|f-k^{\prime}\right\|_{L}$ for $f$ subject to $\mathcal{M}(\tau)$. Maximize $\|f-k\|_{L}=$ $(f-k) \cdot \tau(k)$ subject to $\mathcal{M}(\tau) \cup \mathcal{J}_{k}$. 


\begin{tabular}{|c|c|c|c|c|c|c|c|}
\hline$k$ & 0 & 1 & 2 & 3 & 4 & 5 & 6 \\
\hline Number of Sets & 386 & 2224 & 3704 & 3774 & 4482 & 1698 & 380 \\
\hline$\beta_{k}$ & $\frac{33}{194}$ & $\frac{5}{48}$ & $\frac{5}{71}$ & $\frac{11}{194}$ & $\frac{5}{99}$ & $\frac{291}{6038}$ & $\frac{274}{5969}$ \\
\hline$\gamma_{k}$ & $\frac{1}{4}$ & $\frac{3}{14}$ & $\frac{1}{6}$ & $\frac{5}{38}$ & $\frac{3}{28}$ & $\frac{7}{78}$ & $\frac{4}{57}$ \\
\hline
\end{tabular}

TABLE 1. Kronecker Constants and Sharp $k$-Independence

\begin{tabular}{|c|c|c|c|c|c|c|c|}
\hline $\mathrm{n}$ & 3 & 4 & 5 & 6 & 7 & 8 & 9 \\
\hline$\alpha\{1,2, \ldots, n\}$ & $\frac{1}{4}$ & $\frac{3}{10}$ & $\frac{16}{47}$ & $\frac{49}{134}$ & $\frac{41}{107}$ & $\frac{2}{5}$ & $\frac{12}{29}$ \\
\hline $\begin{array}{c}\text { Previously Best } \\
\text { Known Lower Bound }\end{array}$ & .045 & .139 & .189 & .221 & .243 & .260 & 0.272 \\
\hline
\end{tabular}

TABLE 2. Kronecker Constants for $\{1, \ldots, n\}$

- We exhaust all choices of $\tau$ and subsequent choices of $\mathcal{J}_{k}$. The value of $\alpha(T)$ is the largest maximum occurring in any of the sub-problems.

7.1. Numerical Results. Angular Kronecker constants have been archived at [Hare and Ramsey 2011] for all triples $\{a, b, c\}$ with $0<a<b<c \leq 50$ and $\operatorname{gcd}(a, b, c)=1$. There are 16648 of these triples.

A complete listing of the angular Kronecker constants for relatively prime fourtuples of integers from $\{1, \ldots, 20\}$ can also be found at [Hare and Ramsey 2011].

In Table 1 we summarize our results relating Kronecker constants to the sharp $k$-independence properties. In that table "Number of Sets" is the number of sets examined that were sharp $k$-independent for $k=0, \ldots, 6 ; \beta_{k}=\inf \{\alpha(T): T$ is sharp $k$-independent $\} ; \gamma_{k}=\sup \{\alpha(T): T$ is sharp $k$-independent $\}$. Based on this numerical evidence, we speculate that $\sup \{\alpha(T): T$ sharp $k$-independent $\}$ tends to 0 as $k \rightarrow \infty$.

As well, we ran our algorithm on home and office desktop computers for the sets $\{1,2, \ldots, n\}$. For $1 \leq n \leq 9$ the values are given in Table 2 . Of course, the Kronecker constants must tend to $1 / 2$ as $n \rightarrow \infty$. For comparison, the table includes previously known best lower bounds, which are derived from [Graham and Hare 2006a, Cor. 4.6]. 
[Beyer 1987]

[Galindo and Hernandez 1999]

[Givens and Kunen 2003]

[Graham and Hare 2006a]

[Graham and Hare 2006b]

[Graham and Hare 2008]

[Graham and Lau 2007]

[Graham and McGehee 1979]

[Hare and Ramsey 2011]

[Kahane 1995]

[Kunen and Rudin 1999]

[Lopez and Ross 1975]

[Nemhauser and Wolsey 1988]

[Varopoulos 1968]

\section{REFERENCES}

W. H. Beyer (Ed.) CRC Standard Mathematical Tables, 28th edition, CRC Press, 1987, 123-124.

J. Galindo and S. Hernandez, The concept of boundedness and the Bohr compactification of a MAP abelian group, Fund. Math. 15(1999), 195-218.

B. N. Givens and K. Kunen, Chromatic numbers and Bohr topologies, Top. Appl. 131(2003), 189-202.

C.C. Graham and K.E. Hare, $\varepsilon$-Kronecker and $I_{0}$ sets in abelian groups, I: arithmetic properties of $\varepsilon$-Kronecker sets, Math. Proc. Camb. Phil. Soc. 140(2006), 475-489.

06] C.C. Graham, K.E. Hare and T.W. Korner, $\varepsilon$-Kronecker and $I_{0}$ sets in abelian groups, II: sparseness of products of $\varepsilon$ Kronecker sets, Math. Proc. Camb. Phil. Soc. 140(2006), 491508.

C.C. Graham and K.E. Hare, $\varepsilon$-Kronecker and $I_{0}$ sets in abelian groups, III: interpolation by measures on small sets, Studia Math. 171(2005), 15-32.

C.C. Graham and K.E. Hare, $\varepsilon$-Kronecker and $I_{0}$ sets in abelian groups, IV: interpolation by non-negative measures, Studia Math. 177(2006), 9-24.

C.C. Graham and K.E. Hare, Characterizing Sidon sets by interpolation properties of subsets, Colloq. Math. 112(2008), 175199.

C.C. Graham and A. T-M. Lau, Relative weak compactness of orbits in Banach spaces associated with locally compact groups, Trans. Amer. Math. Soc., 359(2007), 1129-1160.

C.C. Graham and O C. McGehee, Essays in commutative harmonic analysis, Springer-Verlag, 1979.

K.E. Hare and L.T. Ramsey, Upper and lower bounds for Kronecker constants of three-element sets of integers, arXiv:1108.3802v2, 2011.

J-P. Kahane, Algebres tensorielles et analyse harmonique, Seminaire Bourbaki, 1964-6, Exposes 277-312, Soc. Math. France, Paris, 1995, p221-230.

K. Kunen and W. Rudin, Lacunarity and the Bohr topology, Math. Proc. Camb. Phil. Soc. 126(1999), 117-137.

J. Lopez and K. Ross, Sidon sets, Lecture notes in Pure Appl. Math. 13 Dekker, New York, 1975.

G. L. Nemhauser and L. A. Wolsey, Integer and Combinatorial Programming, Wiley-Interscience Series in Discrete Mathematics and Optimization, John Wiley \& Sons, New York, 1988.

N. Varapoulos, Tensor algebras and harmonic analysis, Acta Math. 119(1968), 51-112.

Dept. of Pure Mathematics, University of Waterloo, Waterloo, Ont., Canada, N2L $3 \mathrm{G} 1$

E-mail address: kehare@uwaterloo.ca

Dept. of Mathematics, University of Hawail at Manoa, Honolulu, Hi, USA, 96822

E-mail address: ramsey@math.hawaii.edu 